\title{
Cladistics
}

Cladistics 34 (2018) 517-541

$10.1111 /$ cla. 12225

\section{Combined phylogenetic analysis of the subclass Marchantiidae (Marchantiophyta): towards a robustly diagnosed classification}

\author{
Jorge R. Flores ${ }^{\mathrm{a}, \mathrm{b}, *}$, Santiago A. Catalano ${ }^{\mathrm{a}, \mathrm{b}}$, Jesus Muñoz ${ }^{\mathrm{c}}$ and Guillermo M. Suárez ${ }^{\mathrm{a}, \mathrm{b}}$ \\ ${ }^{a}$ Unidad Ejecutora Lillo (UEL; FML-CONICET), Miguel Lillo 251, S.M. de Tucumán 4000, Argentina; ${ }^{b}$ Facultad de Ciencias Naturales e \\ Instituto Miguel Lillo, Universidad Nacional de Tucumán, Miguel Lillo 205, S.M. de Tucumán 4000, Argentina; ${ }^{c}$ Real Jardín Botánico \\ (RJB-CSIC), Plaza de Murillo 2 Madrid, 28014, Spain
}

Accepted 22 August 2017

\begin{abstract}
The most extensive combined phylogenetic analyses of the subclass Marchantiidae yet undertaken was conducted on the basis of morphological and molecular data. The morphological data comprised 126 characters and 56 species. Taxonomic sampling included 35 ingroup species with all genera and orders of Marchantiidae sampled, and 21 outgroup species with two genera of Blasiidae (Marchantiopsida), 15 species of Jungermanniopsida (the three subclasses represented) and the three genera of Haplomitriopsida. Takakia ceratophylla (Bryophyta) was employed to root the trees. Character sampling involved 92 gametophytic and 34 sporophytic traits, supplemented with ten continuous characters. Molecular data included 11 molecular markers: one nuclear ribosomal (26S), three mitochondrial genes (nad1, nad5, rps3) and seven chloroplast regions (atpB, psbT-psbH, rbcL, $I T S, r p o C 1, r p s 4, p s b A)$. Searches were performed under extended implied weighting, weighting the character blocks against the average homoplasy. Clade stability was assessed across three additional weighting schemes (implied weighting corrected for missing entries, standard implied weighting and equal weighting) in three datasets (molecular, morphological and combined). The contribution from different biological phases regarding node recovery and diagnosis was evaluated. Our results agree with many of the previous studies but cast doubt on some relationships, mainly at the family and interfamily level. The combined analyses underlined the fact that, by combining data, taxonomic enhancements could be achieved regarding taxon delimitation and quality of diagnosis. Support values for many clades of previous molecular studies were improved by the addition of morphological data. The long-held assumption that morphology may render spurious or low-quality results in this taxonomic group is challenged. The morphological trends previously proposed are re-evaluated in light of the new phylogenetic scheme.
\end{abstract}

(C) The Willi Hennig Society 2017.

\section{Introduction}

Among embryophytes (land plants), liverworts (Marchantiophyta) are recognized as the basal group (Qiu et al., 1998; Nickrent et al., 2000; Karol et al., 2001). The complex thalloid liverworts (Marchantiidae) encompass about 345 species and 36 genera (Villarreal et al., 2016) which are remarkably different from other embryophytes. Complex thalloid liverworts are characterized by a combination of both plesiomorphic characters and novelties in their structure. On the one hand, several of these morphological features, such as a

\footnotetext{
*Corresponding author. E-mail address: jrflores@conicet.gov.ar
}

haplodiploid life cycle and the absence of vascular tissue, are widespread among bryophytes. On the other, highly specialized fertile branches (gametangiophores), internal schizogenous air chambers and pegged rhizoids are traits exclusive to the complex thalloid liverworts (Schuster, 1984a,b; Bischler, 1998; Crandall-Stotler and Stotler, 2000). Given their position within embryophytes and the morphological peculiarities, Marchantiidae stand as crucial taxa to disentangle the evolutionary history of land plants.

The first classifications within Marchantiidae, based on phylogenetic systematic principles, were presented based on morphological data. However, the subsequent classifications relied exclusively on molecular data. The most comprehensive morphological phylogeny (Bischler, 
1998) supported the distinction of a basal Ricciineae and a derived group comprising Corsiineae + Targioniineae + Marchantiineae. One of the few combined analyses was published by Boisselier-Dubayle et al. (2002) and included 27 out of 35 genera of Marchantiidae. The combined trees obtained in that study rendered a completely different pattern of relationships from that published by Bischler (1998). Subsequent molecular analyses largely agree with the relationships obtained by Boisselier-Dubayle et al. (2002), except in the relationships among morphologically simple families (Wheeler, 2000; Crandall-Stotler et al., 2005; Forrest et al., 2006; HeNygrén et al., 2006).

Boisselier-Dubayle et al. (2002) analysed the conflict between morphological data and molecular data, showing that both sources of evidence produced very different trees, but also that support values could be improved by concatenating different data types. Crandall-Stotler et al. (2005) reported a high degree of homoplasy within morphological data. Nevertheless, they were able to find synapomorphies for most of the nodes. Subsequently, many diagnoses were modified and several more diagnostic characters were found to define higher taxonomic groups (e.g. lenticular apical cell for Metzgeriidae; Crandall-Stotler et al., 2009) than in previous classifications. Contrasting with Boisselier-Dubayle et al. (2002) and Crandall-Stotler et al. (2005), He-Nygrén et al. (2006) did not carry out an explicit survey on character conflict. Even so, they performed the most extensive combined analysis in terms of morphological data inclusion. In addition, the results obtained by He-Nygrén et al. (2006) displayed no significant differences when different optimality criteria were considered. Although He-Nygrén et al.'s study was the first attempt to reach a reliable diagnosed classification, Marchantiidae taxonomic sampling included only 13 genera (37\% of the accepted genera in the subclass).

The current accepted classification (Crandall-Stotler et al., 2009) was achieved as a result of several multilocus phylogenies produced during the last decade. Compared to the previous morphology-based classification, several taxonomic categories were omitted. In addition, many genera of Marchantiidae were relocated in completely different categories (e.g. Monocarpus D.J. Carr (Forrest et al., 2015) or Neohodgsonia (Perss.) Perss. (Crandall-Stotler et al., 2009)). As Crandall-Stotler et al. (2009) pointed out, diagnosing these unexpected groups - from the morphological point of view-represented a real challenge.

Despite the advances in the morphological knowledge of Marchantiidae accomplished in the last decade (e.g. Duckett et al., 2014), there was no attempt to include this new information into quantitative phylogenetic analyses. Although recently performed phylogenies sampled a considerable number of molecular markers (Forrest et al., 2006, 2015; Villarreal et al.,
2016), support values for several nodes were still low. On the one hand, some taxonomic changes have been recently proposed based upon these clades with low support (Long et al., 2016a,b). For example, given that the genera Exormotheca Mitt. and Stephensoniella Kashyap were found to be the sister taxa of Cronisia Berk., Exormothecaceae was merged with Corsiniaceae (Long et al., 2016a). However, the node constituted by Cronisia, Exormotheca and Stephensoniella lacked significant support values (fig. 2 in Villarreal et al., 2016). On the other hand, taxonomic proposals based on nodes with high support were controversial from a morphological perspective (Forrest et al., 2015; Long et al., 2016b). Even if the average support at the family level was high in recent molecular studies (Forrest et al., 2006; Villarreal et al., 2016), deep nodes remained ambiguous (Wheeler, 2000; BoisselierDubayle et al., 2002; Forrest et al., 2006; Villarreal et al., 2016). A large number of the inclusive nodes within Marchantiales, the crown group of the subclass (Crandall-Stotler et al., 2009), still had low support values (Villarreal et al., 2016). In particular, derived nodes (Ricciaceae, Oxymitraceae, Monocleaceae, etc.) were hard to resolve even in combined analyses (Boisselier-Dubayle et al., 2002). Therefore, with few exceptions, interfamily relationships persisted as a taxonomic challenge (Crandall-Stotler et al., 2009).

The strategies to overcome environmental stress and their significance to bryophytes evolution were regular issues of interest in previous studies (Bischler and Jovet-Ast, 1981; Longton, 1988a; Hedderson and Longton, 1996; Bischler, 1998). Bischler (1998) defined four different groups based on different morphological and ecological characteristics, postulating a strong correspondence of those groups with morphological characters and taxonomic/phylogenetic membership. Similar morphologies were explained as consequences of common ancestry rather than similar selective forces (Bischler, 1998; Bischler et al., 2005). Concerning mosses, Longton (1988b) stated that the basic life strategy was being perennial, considering ephemeral strategies as derived. In contrast, Bischler (1998) considered ephemeral life traits as ancestral for complex thalloid liverworts. Although character mapping has been considered to elucidate the evolutionary pattern of some traditional characters (Villarreal et al., 2016), Bischler's hypothesis on the concerted evolution of life-history traits has not been challenged analytically.

The recent modifications within the phylogeny of Marchantiidae have involved both new scenarios of evolutionary trends and changes in morphological diagnosis of different clades within this group. Here we present the results of the largest combined phylogenetic analysis in Marchantiidae in terms of taxon and character sampling. This study allows the solving of previous uncertainties in some nodes, updating group 
diagnoses and testing previous ideas about character evolution. In addition, the contribution to and conflict between morphological characters from the different life phases were evaluated in depth.

\section{Materials and methods}

\section{Taxonomic and morphological character sampling}

Despite taxonomic changes having been recently proposed for some groups, the main taxonomic scheme followed throughout this study was that of Crandall-Stotler et al. (2009). After Long et al. (2016a, b), the genera Exormotheca and Aitchisoniella Kayshap were considered members of Corsiniaceae and Cleveaceae, respectively. However, in order to test recently proposed synonyms (Long et al., 2016a), the following genera were scored as independent entities: Stephensoniella and Exormotheca, and Preissia Corda, Bucegia Radian and Marchantia L. In the combined dataset, ingroup sampling consisted of 37 genera of Marchantiopsida (35 genera of Marchantiidae plus two genera of Blasiidae), which represented all of the orders, families and genera within the class. Outgroups included 15 species of Jungermanniopsida, with the three major subclasses of this group being represented (Jungermaniidae, Pelliidae and Metzgeriidae). In addition, the dataset included species from the three genera of Haplomitriopsida. Takakia ceratophylla (Mitt.) Grolle (Bryophyta) was employed to root the tree. Five genera were absent in the morphological partition: Cavicularia Steph. (Blasiidae), Riella Mont. (Marchantiidae), Apotreubia S. Hatt. \& Mizut. (Haplomitriopsida), Pleurozia Dumort and Pallavicinia Gray (Jungermanniopsida). All but five species were scored mainly from observed specimens: Athalamia pinguis Falc., Austroriella salta J. Milne \& Cargill, Geothallus tuberosus Campb. (Marchantiidae), Pellia epiphylla L. Corda and Lejeunea cavifolia (Ehrh.) Lindb. (Jungermanniopsida). In total, the combined dataset comprised 56 species; 20 more taxa than the last combined analysis (Boisselier-Dubayle et al., 2002). Taxa and vouchers are listed on Table 1.

The morphological dataset comprised 126 characters from different sources. The present morphological matrix was initially constructed by extending that of Bischler (1998). Some characters scored at the phylum level by Crandall-Stotler and Stotler (2000) were also included. The original coding of nine of these "traditional" characters were modified (see Character Definition in the Supplementary material). Along with these traits, 66 completely novel characters were incorporated. Pegged rhizoids, for instance, were recently studied in depth by Duckett et al. (2014). Most of these characters, especially those that were now re-interpreted, were included in a phylogenetic matrix for the first time. Similarly, characters derived from spores have been studied extensively in several taxa (Gupta and Udar, 1986) but infrequently used for phylogenetic analyses within this group; novel data from spores of Marchantiidae were considered in this study. Features representing continuous traits have seldom been considered in phylogenetic analyses of bryophytes. In cases where this sort of information was included, characters were commonly discretized losing valuable phylogenetic information (Farris, 1990; Goloboff et al., 2006). In the present study, 10 different variables were analysed as continuous characters (Goloboff et al., 2006). In order to incorporate the morphological variation among populations, a minimum of 10 specimens per species was examined. The final range of variation for each character was established as the mean value \pm standard deviation (SD). Ninety-two characters were scored from the gametophytic phase and 34 from the sporophyte. In conjunction, these included 12 cytological characters, nine developmental features, 18 sexual traits and 87 macroscopic characters. The morphological dataset presented 4540 more cells than the most extensive morphological dataset at the subclass level to date (1886 cells; Bischler, 1998). A detailed description of character, state definition and images can be found in the Supplementary material. The final dataset is available at Morphobank (Project: 2674; morphobank.org/permalink/?P2674).

\section{Molecular data}

The previous most comprehensive molecular sampling was carried out by Villarreal et al. (2016). The present study focused on extending outgroup sampling and filling gaps within the former analysis. Therefore, $p s b A$ and $r b c L$ markers were sequenced for three species (Plagiochila sp. (Dumort) Dumort; Radula voluta Taylor ex Gottsche, Lindenb. \& Nees and Riccia sp. L.). Amplifications were carried out at the Real Jardín Botánico de Madrid (Spain) following the conditions described by Forrest and Crandall-Stotler (2004; primers described in Table S1). Sequencing was conducted by Macrogen Inc. (Seoul). Sequences by Villarreal et al. (2016) were downloaded from GenBank. Nucleotides were aligned with MAFFT (Katoh and Toh, 2008) using default penalty settings. Ambiguously aligned positions were excluded from the analysis. Data were compiled using GB2TNT (Goloboff and Catalano, 2012), a pipeline to build molecular datasets from GenBank.

Phylogenetic analyses, constrained searches and topology comparisons

Although molecular data are commonly analysed by following model-based methods, the study of 


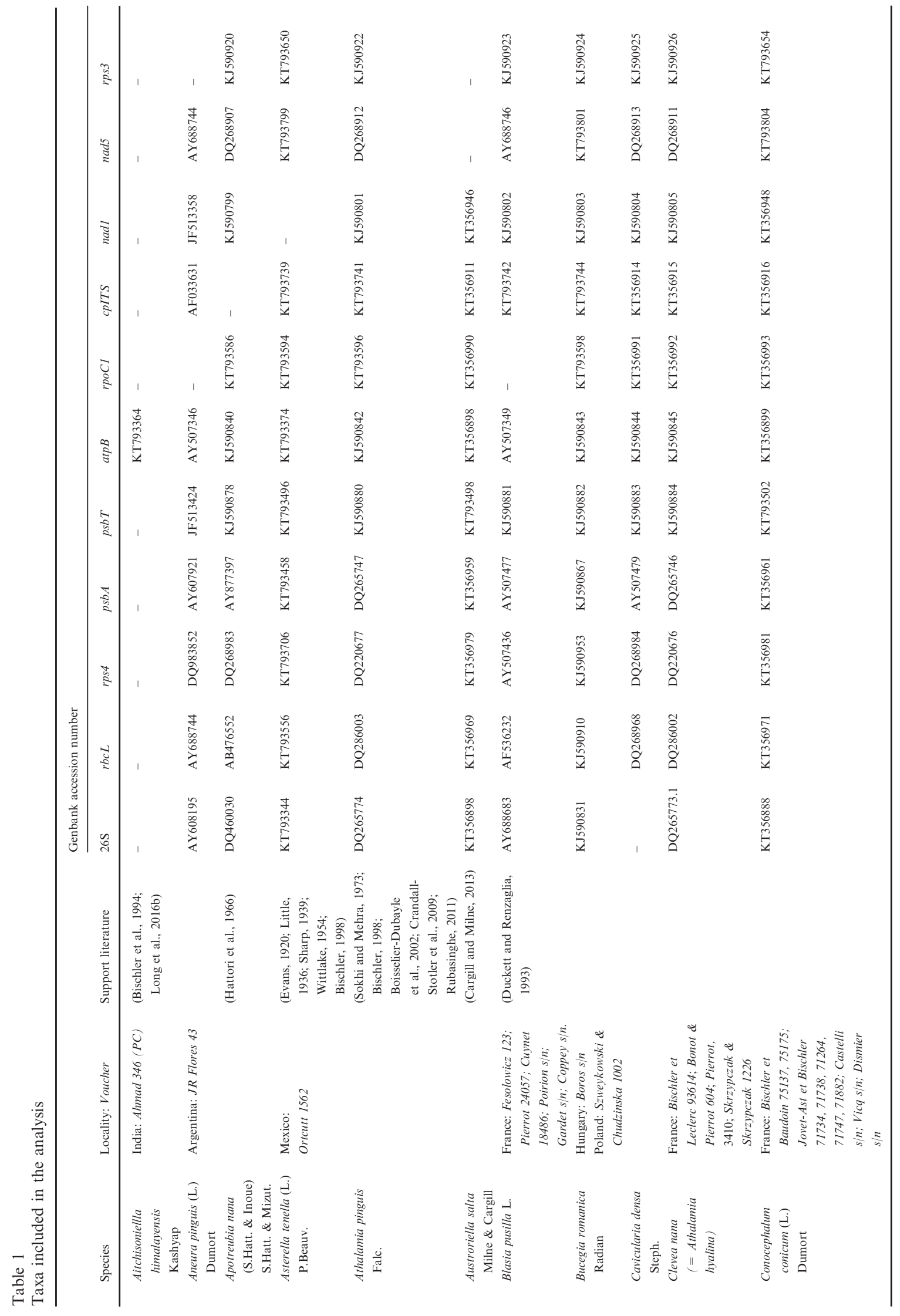




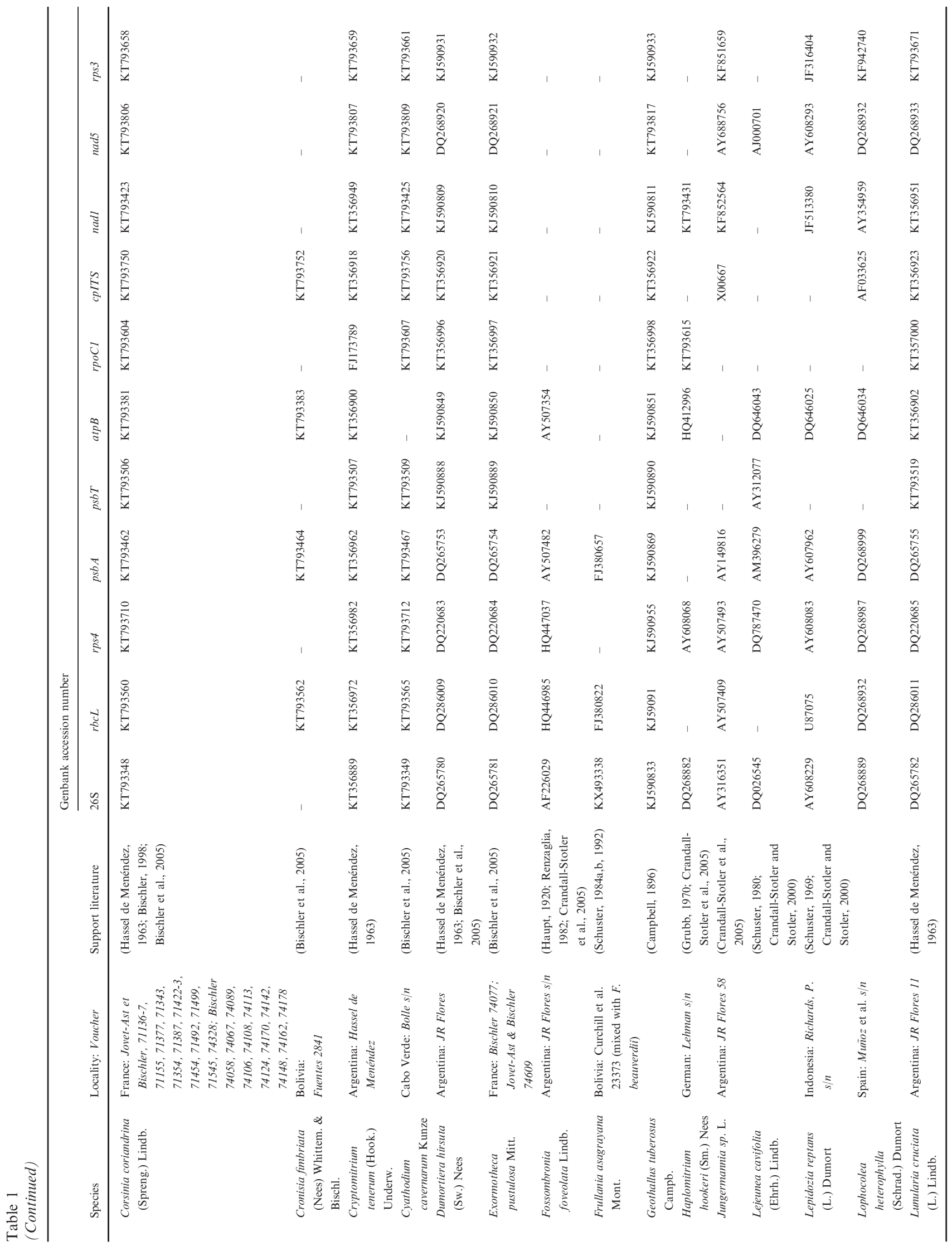




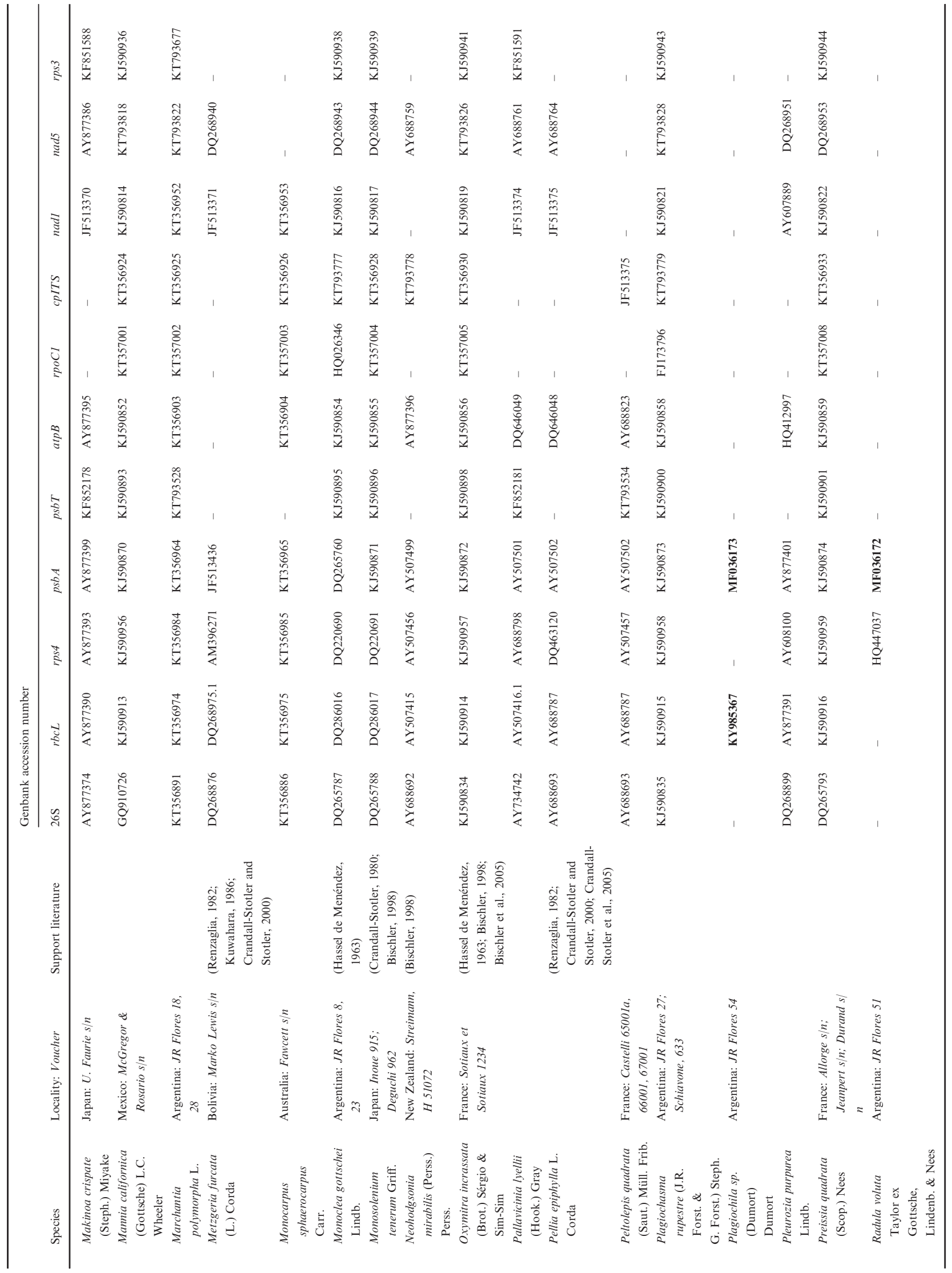




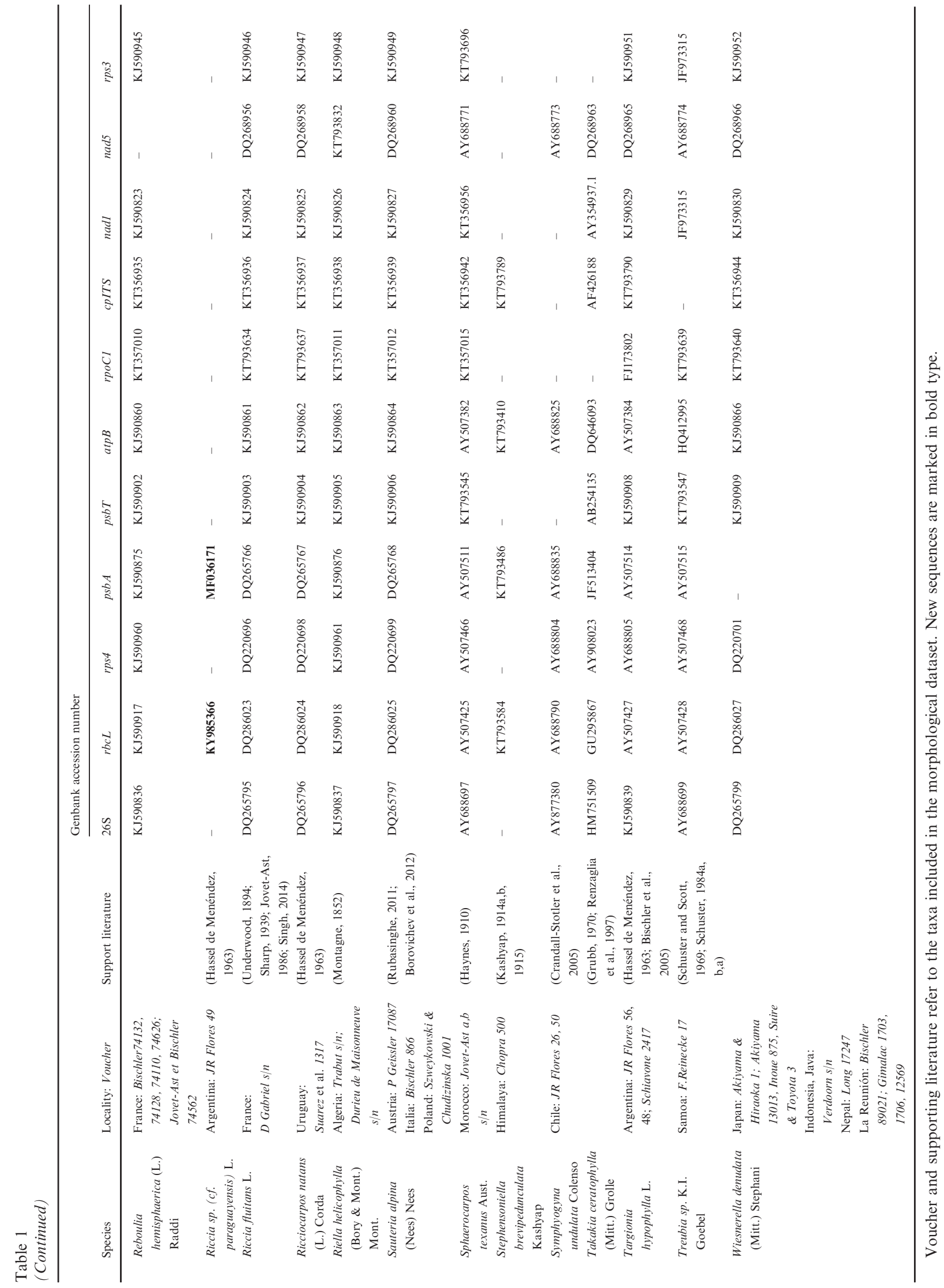


morphology has been based largely on parsimony. In contrast with molecular data, the use of probabilistic models to evaluate morphology has continually led to undesirable results (Goloboff and Pol, 2005; Beck and Lee, 2014; Goloboff et al., 2017). For instance, Mk models have recently been shown to be particularly sensitive to the high heterogeneity rate present in morphological datasets, resulting in spurious groupings and imprecise dating (Steiper and Seiffert, 2012; Beck and Lee, 2014). These outcomes still generate a great reluctance amongst many authors to adopt models as a mean to analyse morphological data. Weighted parsimony, however, has been shown to improve the analysis of morphological data (Goloboff et al., 2008a,b) and has acquired a key role in combined phylogenetic analyses (e.g. Mirande, 2017). As the principal premise of this paper is to achieve a phylogenetic hypothesis based on a total-evidence approach, searches were performed under weighted parsimony as the optimality criterion.

Therefore, the main systematic and taxonomic conclusions of the present study are derived from the analysis of the combined dataset under extended implied weighing, weighting blocks in accordance to their average homoplasy (Goloboff, 2014). The blocks represented each gene and the morphological dataset.

Group sensitivity and searches. In order to assess group stability, searches were conducted across several weighting schemes for individual partitions and the combined dataset. In addition to the searches under block weighting (BW), an alternative search strategy involved weighting each character according to its homoplasy but taking into account the proportion of missing entries $\left(\mathrm{IW}_{\mathrm{M}}\right)$. In $\mathrm{IW}_{\mathrm{M}}$, each missing entry was assumed to have half of the homoplasy of the observed entries $(P=0.5)$. In searches under both $\mathrm{IW}_{\mathrm{M}}$ and $\mathrm{BW}$, the TNT default concavity value was used $(K=3 ; K 3)$. Three concavity values for standard implied weighting were also explored (K3, K5 and K7; Goloboff, 1993). Finally, the data also were analysed under equal weighting (EqW). All weighting settings were applied to the individual partitions and the combined data (except BW in the case of the morphological dataset). Hence, a total of 17 different analyses were conducted (six for combined data, six for the molecular dataset and five for morphological dataset). In all cases, analyses were carried out in TNT 1.5 (Goloboff et al., 2008a,b; Goloboff and Catalano, 2016) with new technology searches using 10 RAS as starting point. For each replicate, 10 iterations of Ratchet (Nixon, 1999) and Tree Drifting (Goloboff, 1999) were performed, ending the search when after the best score was hit seven times. Support values were estimated by Symmetric Resampling (SR) considering the difference between the most frequent group and the most frequent contradictory group (GC; Goloboff et al., 2003). Additional estimates of support (Bootstrapping and Bremer) were also calculated and are available in Fig. S1.

Topological comparisons. In order to assess the contribution of each source of information to the final result, the topologies recovered from different data types were compared in terms of (i) SPR (subtree pruning and regrafting) distance (Goloboff, 2008) and (ii) number of shared nodes. As a single SPR rearrangement may involve a movement of a few or several nodes, each movement was weighted by a constant value of 5 (Goloboff, 2008). The morphological trees obtained under each weighting condition were compared to all the molecular trees. In turn, the molecular trees were compared to the combined tree. The number of SPR moves and the number of shared nodes were also calculated for the morphological trees obtained by Bischler (1998) and Boisselier-Dubayle et al. (2002). In addition, the same comparison was performed to discern the relative contribution of gametophyte and sporophyte characters. To compare our morphological tree with Bischler (1998) and Boisselier-Dubayle et al. (2002) trees, evaluations involved two different trees as reference: (a) our molecular tree obtained under BW and (b) Villarreal et al.'s (2016) molecular tree. Regarding our two sets of morphological characters, comparisons only used our molecular tree as reference.

Constrained searches. In order to quantify the suboptimality of the groups not retrieved in the best trees, constrained searches were carried out in the combined data under BW. Understanding suboptimality in terms of fit (F) is hardly intuitive. Therefore, the fit difference between the constrained and the unconstrained trees was translated into the number of mean homoplastic characters with an extra step required to explain the suboptimal tree $\left(\bar{x}_{+1}\right.$; Carrizo and Catalano, 2015). The estimation of character with mean homoplasy ( $\bar{x}$ was obtained as the tree length divided by the number of characters. The ratio between the topology fit differences $\left(F_{\text {unconstrained }}, \mathrm{F}_{\text {constrained }}\right)$ and mean character fit differences $\left(\bar{x}, \bar{x}_{+1}\right)$ allowed suboptimality to be conceived in terms of the number of $\bar{x}_{+1}$ $\left[\left(\mathrm{F}_{\text {unconstrained }}-\mathrm{F}_{\text {constrained }}\right) /\left(F_{\bar{x}}-F_{\bar{x}+1}\right)\right]$.

\section{Character reconstruction, synapomorphies and diagnosis}

Group diagnoses were determined by mapping morphological characters onto the combined tree obtained under BW. In addition, the role of different sets of characters to diagnose specific clades was addressed. 
Because sporophytic characters have commonly been viewed as having no taxonomic information at lower levels (Schuster, 1984a,b; Crandall-Stotler and Stotler, 2000; Crandall-Stotler et al., 2009), special attention was paid to those characters. In particular, the proportion of synapomorphies derived from sporophytic characters was quantified for each node. To assess whether sporophytic characters diagnose taxonomic groups below the class level, the number of sporophytic synapomorphies per taxonomic category was also measured.

Phylogenetic searches, sensitivity assessment and diagnosis evaluation were implemented in scripts using TNT macro language. These are available upon request.

\section{Life history patterns and taxonomic membership}

Bischler (1998) hypothesized that life-history traits were phylogenetically correlated in Marchantiidae and, consequently, morphological similarity was caused by common ancestry. In order to test these hypotheses in light of the new data, the four patterns found by Bischler (named Groups I-IV) and the putative associated characters were mapped onto the final tree. Given their relevance in liverwort biology (Bischler et al., 2005), four features were optimized: branch length, number of spores per capsule, spore size and capsule dehiscence. Branch length and spore size were scored as continuous characters; capsule dehiscence was scored as a binary character (cleistocarpous/noncleistocarpous). The number of spores per capsule was scored as a multistate character: less than 500 spores (0), more or equal to 1000 and less than 10000 (1) and more than 10000 spores (2).

\section{Results}

\section{Combined Tree under BW and Constrained Searches}

The combined dataset analysed under extended implied weighting (BW) produced a single fully resolved tree (Fig. 1). It was largely congruent with recent phylogenetic analyses (Forrest et al., 2015; Long et al., 2016a,b).

The major groups (Haplomitriopsida, Jungermanniopsida and Marchantiopsida) were recovered as monophyletic with high support values $(\approx 100$; Fig. 1$)$, as well as the sister relationship among the four orders within Marchantiidae. Some of these nodes had already been found in previous studies but have remained without a formal recognition (Crandall-Stotler et al., 2009; Forrest et al., 2015). To facilitate descriptions, some of these nodes are referred to as clades A-F. Clade A was rendered as the association between the genus Monocarpus and the order Sphaerocarpales (Sphaerocarpus, Geothallus, Austroriella and Riella) with strong evidence (SR 98,
Fig. 1); the monophyly of Sphaerocarpales s.s. received more moderate support (SR 72). As in recent molecular phylogenies (Forrest et al., 2015; Villarreal et al., 2016), the previous definition of Marchantiales was not supported. Clade B was established upon the node constituted by Lunularia (Lunulariales) and the remaining genera of Marchantiales expect Monocarpus. This clade with a moderate (to high) support value (SR 88), was recovered as the sister group to Clade A.

Mid-level nodes within Clade B were recovered in most cases with low support. The node F (Riccia + Ricciocarpos + Oxymitra) + E (Monoclea + Conocephalum) was the sister clade to the monophyletic Aytoniaceae (Asterella + Cryptomitrium + Mannia + Plagiochasma + Reboulia). The poorly supported clade Monosolenium + Cyathodium was related to the highly supported Clade D (Exormotheca + Aitchisoniella + Stephensoniella + Corsinia + Cronisia). Clade C (Wiesnerella + Targionia) was sister to both the remaining morphologically simple groups and Aytoniaceae (Fig. 1). In contrast, the less inclusive nodes between families tended to be well supported. Clades C, D, F and $\mathrm{E}$ received support values from 82 to 100 (Fig. 1).

At the family level, Cleveaceae and Corsiniaceae (Long et al., 2016a) were both nonmonophyletic because Aitchisoniella was nested within the latter group. The genera Cronisia and Corsinia formed a moderately supported clade (SR 76; Fig. 1) with Exormotheca as their sister taxa (SR 64; Fig. 1). Stephensoniella constituted a highly supported clade with Aitchisoniella (SR 100; Fig. 1). The clade formed by Sauteria, Clevea, Peltolepis and Athalamia was also highly supported (SR 100; Fig. 1). The other nonmonotypic families were retrieved as monophyletic with general high support.

Phylogenetic searches forcing the monophyly of groups absent from the best trees led to widely different suboptimality values depending on the considered taxa. On the one hand, ingroup families were highly suboptimal regarding the BW combined tree. The monophyly of Corsiniaceae entailed a difference of 167.1 mean homoplastic characters gaining a further step. As for Cleveaceae, its monophyly involved 172.2 mean characters gaining an extra step. Comparatively, outgroup taxonomic groups implied less than one step in a character with mean homoplasy $(0.2-0.6)$. Therefore, there was no evidence to discard the monophyletic status of outgroup taxa whereas the monophyly of the ingroup families was considerably questioned.

\section{Partitioned analyses}

Molecular dataset. The strict consensus of the molecular trees recovered across the entire analytical conditions had 34 nodes (out of 54; Fig. 2). Molecular trees recovered Jungermanniopsida and Marchantiopsida as monophyletic. Inside 

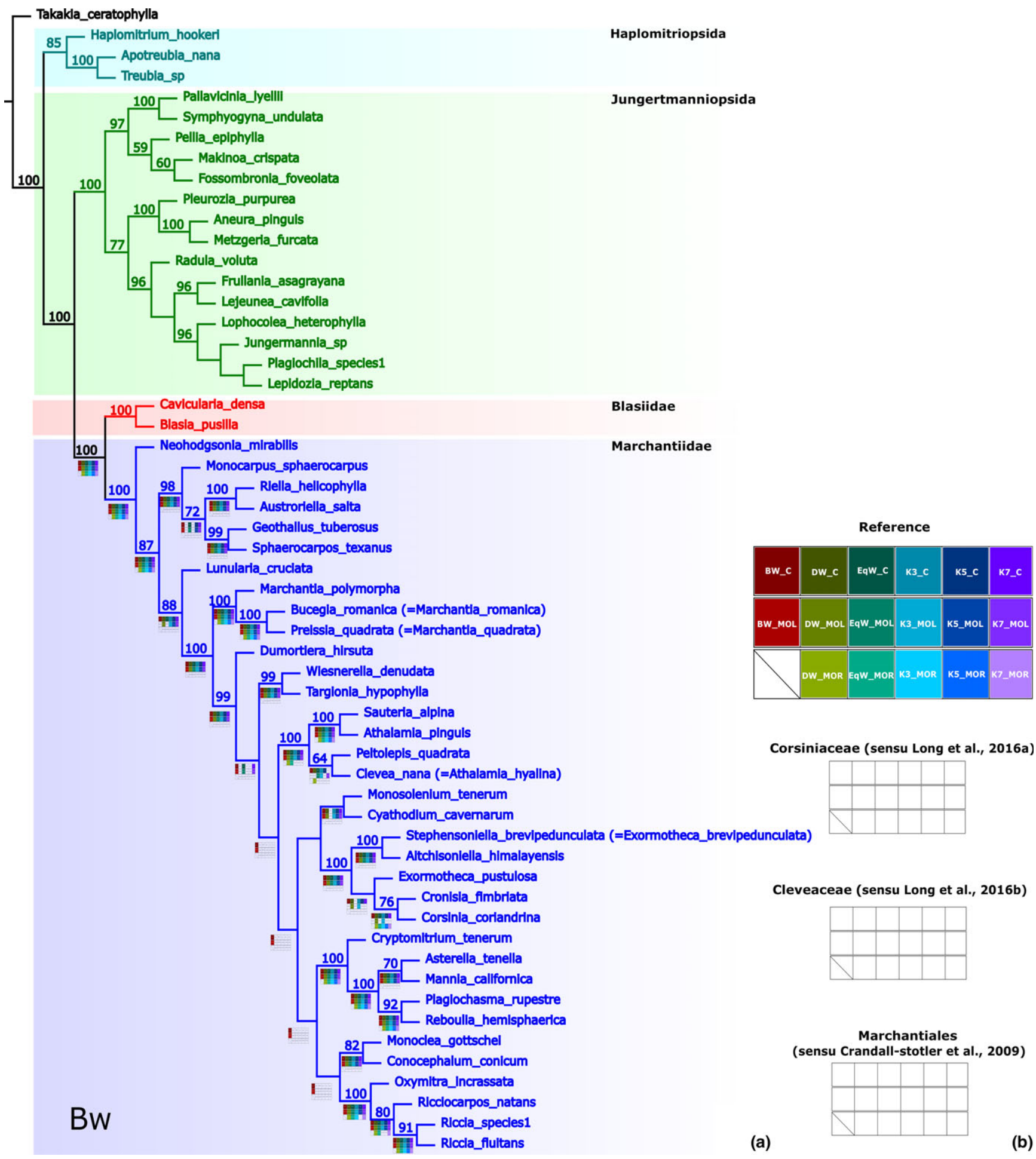

Corsiniaceae (sensu Long et al., 2016a)

Cleveaceae (sensu Long et al., 2016b)

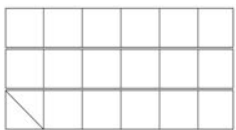

Marchantiales

(sensu Crandall-stotler et al., 2009)

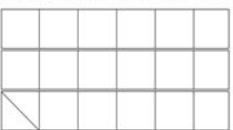

Fig. 1. (a) Combined tree under extended implied weighting (Block weighting). Symmetric Resampling values are indicated above branches (only $>50$ values are shown; see additional support measures in the Fig. S1 in the Supplementary material). Sensitivity plots for ingroup nodes monophyly are shown below branches. (b) Sensitivity plots for Cleveaceae, Corsiniaceae and Marchantiales [as defined by Long et al. (2016a,b) and Crandall-Stotler et al. (2009)], two groups that were not monophyletic in the combined analysis under block weighting. Sensitivity plot reference: coloured squares represents monophyly. $\mathrm{C}=$ combined data, $\mathrm{MOL}=$ molecular data, $\mathrm{MOR}=$ morphological data. Extended implied weighting settings: block weighting (BW) and character weighted considering their homoplasy and number of missing entries (IWM). K3, K5 and K7 are concavities values explored under standard implied weighting. EqW refers to equal weighting. [Colour figure can be viewed at wileyonlinelibrary. com]. 


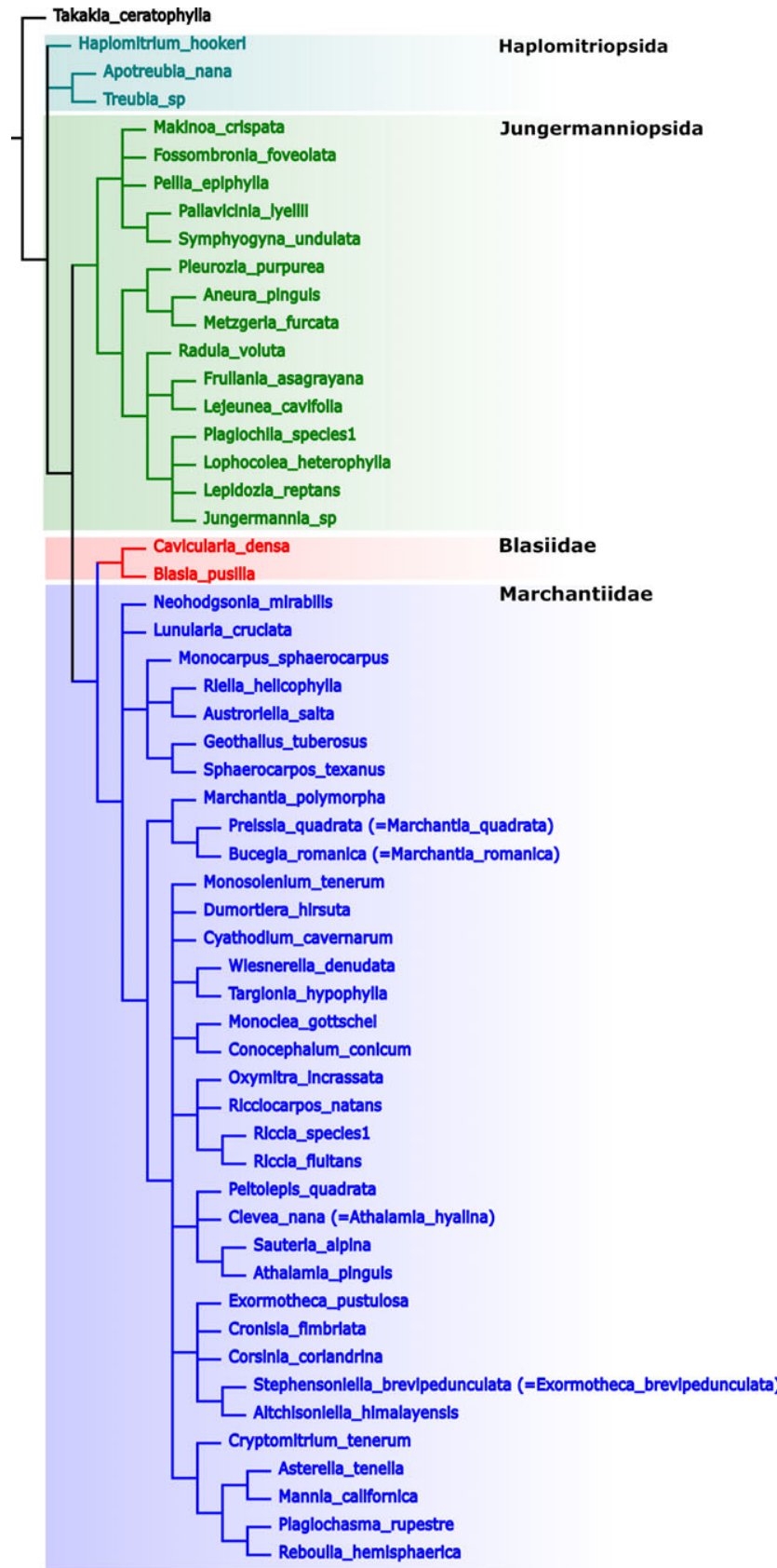

Molecular trees strict consensus

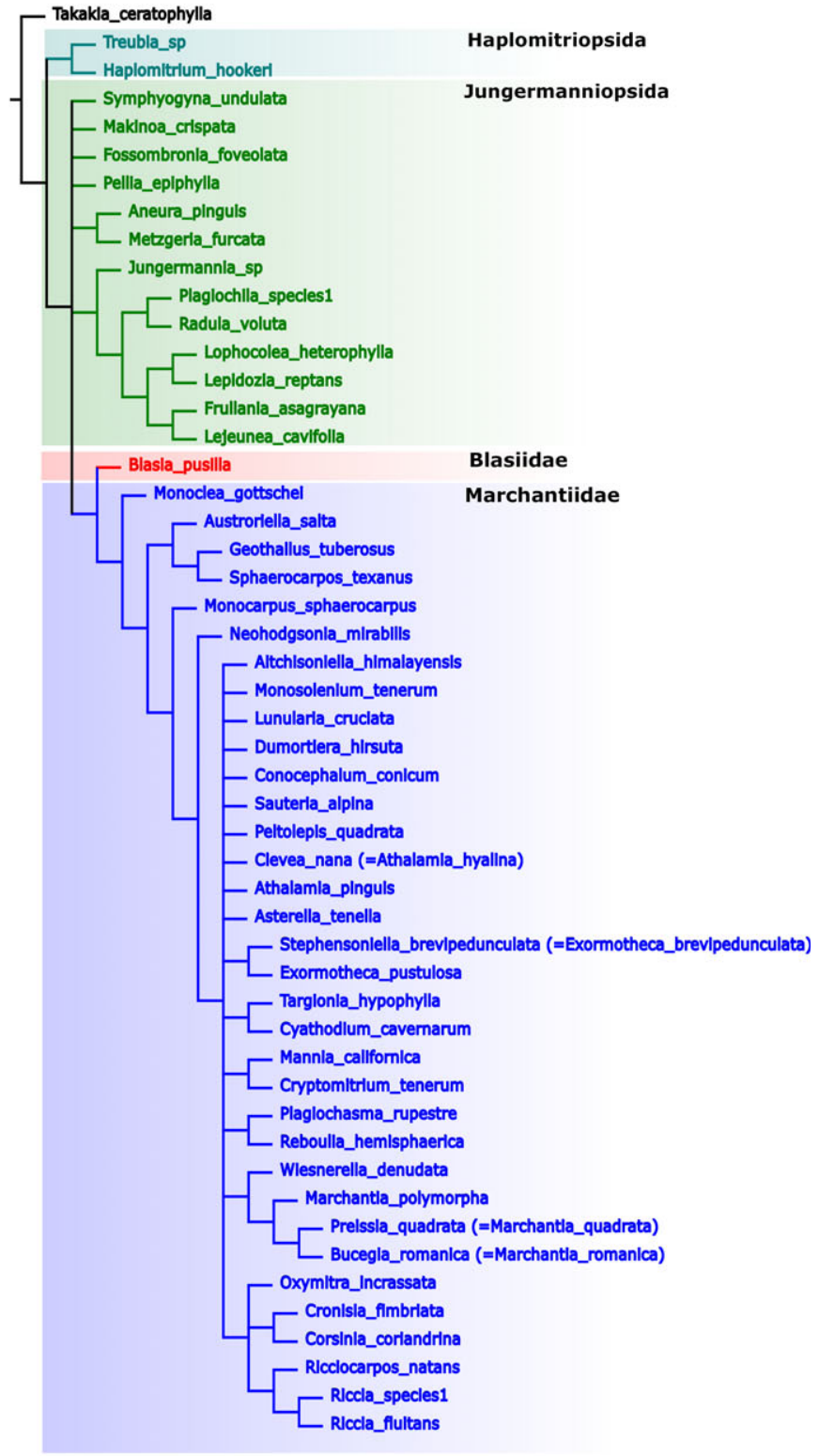

Morphological trees strict consensus

Fig. 2. Strict consensus of the molecular (left) and morphological trees (right) obtained under the different weighting schemes. [Colour figure can be viewed at wileyonlinelibrary.com].

Marchantiopsida, relationships were poorly resolved. The association between the orders Lunulariales or Neohodgsoniales, and the remaining orders was unresolved. The family Marchantiaceae was sister to the remaining Marchantiales. The rest of the families within Marchantiales were placed in a nine-way polytomy. Clades with high support values in the combined tree under BW were also recovered in the analysis of the molecular partition. The genera Aitchisoniella and
Stephensoniella were sister taxa within a clade constituted by the remaining genera of Corsiniaceae. Cleveaceae was not recovered as monophyletic. Searches under BW concluded in a single fully resolved tree, highly similar to the combined tree (see below under Relative data contribution).

Morphological dataset. The strict consensus of the morphological trees obtained across the entire 
analytical conditions had 29 nodes (out of 49; Fig. 2). The order Marchantiales (in the sense of CrandallStotler et al., 2009) was not recovered as monophyletic. Monoclea was found to be closely related to Sphaerocarpales and Lunularia was nested within Marchantiales. Monocarpus was the sister taxon of the remaining Marchantiales. Neohodgsonia was not recovered inside Marchantiaceae. The rest of the taxa were placed within a 16-way polytomy. The genus Aitchisoniella was not grouped with the rest of the family Cleveaceae although its association with other families was unclear. The other members of Cleveaceae did not constitute a monophyletic group. Corsiniaceae was also nonmonophyletic. The genera Exormotheca and Stephensoniella were sister taxa whereas Corsinia + Cronisia were related to Ricciaceae and Oxymitraceae. Searches under standard implied weighting (K7) led to a single fully resolved tree which maximized congruence with both the combined data and the molecular data (see below under Relative data contribution).

\section{Sensitivity analysis}

About two-thirds of the ingroup clades (22 of 36) were not affected by different weighting schemes (Fig. 1). Although it was not a strict pattern, clades with high support values tended to be less sensitive to parameter variation. Unsupported nodes $(\mathrm{SR}<50)$ were nonmonophyletic in 11 to 15 weighting schemes. Weakly to moderately supported clades (SR 50-90) were not monophyletic in two to seven weighting schemes. Highly supported groups $($ SR $>90)$ were not monophyletic in five weighting conditions or less. Two exceptions to this pattern were Sphaerocarpales (SR 72) and Oxymitraceae + Ricciaceae (SR 100), which were nonmonophyletic in nine analytical conditions. The genus Exormotheca behaved as a wildcard taxon, alternating its position with members of Corsiniaceae. The most inclusive nodes within Marchantiales (i.e. excluding Marchantiaceae and Dumortieraceae) were extremely sensitive. Moreover, such nodes were only recovered in two analytical conditions (molecular and combined data under BW; SR < 50). However, lower level clades within Marchantiales were recovered under different weighting schemes. The clades Monocleaceae + Conocephalaceae, Exormothecaceae + Corsiniaceae and Monosoleniaceae + Cyathodiaceae were markedly stable (10 to 12 schemes). The genus Aitchisoniella constituted a stable clade with Stephensoniella in 12 weighting schemes.

Nodes of the morphological trees were more sensitive to parameter variation than molecular trees (sensitivity plots in Fig. 1). Five families, and relationships inside them, were resolved by the morphological data (Ricciaceae, Aytoniaceae, Corsiniaceae, Cleveaceae and Marchantiaceae). Taxa relationships inside Sphaerocarpales were considerably more stable in the molecular data.

\section{Relative data type contribution}

Trees derived from molecular data were highly similar to the BW combined tree. In particular, the BW molecular tree was the most similar to the BW combined cladogram (1.2 SPR; Fig. 3). The molecular tree obtained under BW also shared 48 nodes with the combined tree. Among morphological trees, the tree obtained considering a concavity value of seven (K7) was the most similar to the BW molecular tree $(8.1$ SPR moves and 17 shared nodes).

Regardless of the reference tree (our BW molecular tree or Villarreal et al.'s (2016) tree), the comparisons between the K7 morphological tree and Bischler (1998) and Boisselier-Dubayle et al. (2002) trees yielded lower SPR values for our morphological tree (Table 2). Bischler's (1998) morphological tree involved 9.1 and 9.2 SPR moves to the BW molecular tree and Villarreal et al.'s (2016) tree, respectively. Boisselier-Dubayle et al.'s (2002) tree entailed 9.5 SPR moves to the BW molecular tree and 10.7 SPR moves to Villarreal et al.'s (2016) tree.

In terms of shared nodes, values varied depending on the reference tree (Table 2). The number of shared nodes was slightly higher for Bischler's tree when our BW molecular tree was used as reference (18 shared nodes for Bischler's tree; 17 shared nodes for the K7 tree and Boisselier-Dubayle et al.'s tree). In contrast, the number of shared nodes was higher for the $\mathrm{K} 7$ morphological tree when Villarreal et al.'s tree was used as reference (18 shared nodes for our K7 tree; 17 shared nodes for Bischler's and Boisselier-Dubayle et al.'s trees).

The gametophyte tree was more similar to the BW molecular tree than the sporophyte tree. In terms of shared nodes, the single gametophytic tree had 15 common nodes with the molecular tree and an SPR distance of 8.38 weighted movements (Fig. 4). The analysis including only sporophytic characters produced 100 optimal trees which shared eight nodes with the molecular tree and had an average SPR distance of 12.8 weighted movements.

Conflict and contribution of data sources were also reflected in the support values. Despite the wide differences among molecular trees and morphological trees, average support values were improved in the combined analysis in comparison with the molecularonly analysis. In particular, five clades of the ingroup had higher support values (Figs 2 and 4). The mean SR support value was 70 for the BW combined tree, whereas it was 65.7 for the BW molecular topology. 


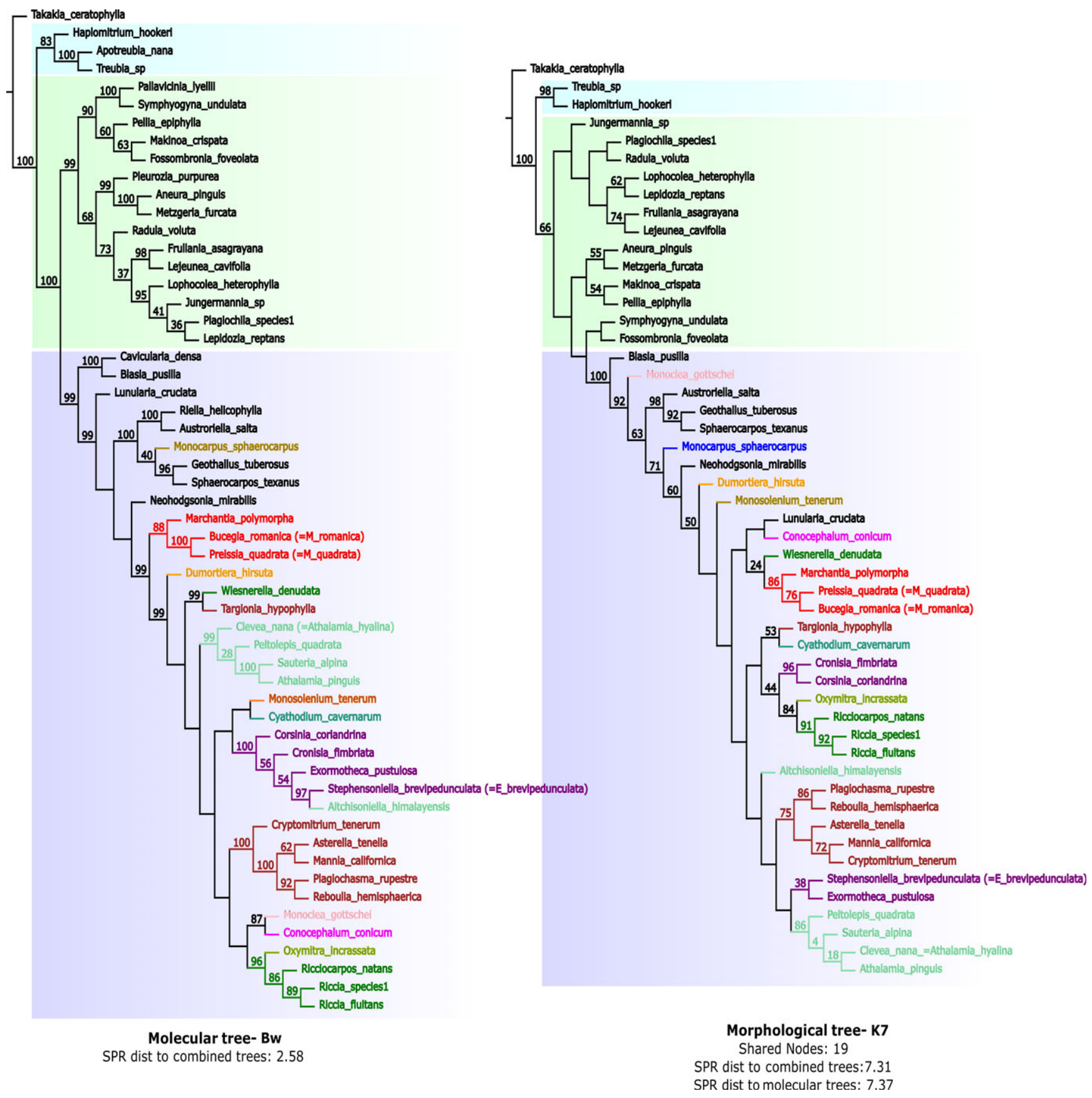

Fig. 3. Molecular tree under extended implied weighting (Block weighting) and morphological tree under implied weighting $(K=7)$ which maximised similarity among data types. Weighted SPR movements to the reference tree and shared nodes are indicated below each topology. [Colour figure can be viewed at wileyonlinelibrary.com].

\section{Synapomorphy mapping and diagnosis assessment}

Mapping morphological characters on the combined tree allowed determination of synapomorphies for 26 out of 35 ingroup nodes. The class Marchantiopsida and seven high-level clades inside it had high to moderate support values and morphological apomorphic characters (Fig. 5; Table 3). Marchantiopsida and Marchantiidae were both supported by nine synapomorphies whereas node B had 10 synapomorphic characters (being diagnosed for the first time). Clade F was supported by seven synapomorphies; two continuous characters and five discrete characters. The Clade E was supported by a single spore trait, germ tube absent. Two characters were diagnostic for Clade D. Three characters were synapomorphies for both clades C and A. Descriptions of these specific groups are in Table 3, a detailed list of 
Table 2

Comparison among different morphology-based trees (our morphological tree obtained under implied weighting, and Bischler's and BoisselierDubayle et al.'s trees). The tree obtained in the present study under K7 implied weighting maximized similarity with reference trees in three out of four cases (bold). Bischler's tree maximized the number of shared nodes with the BW molecular tree (bold)

\begin{tabular}{lllll}
\hline & $\begin{array}{l}\text { SPR moves to the } \\
\text { BW molecular tree }\end{array}$ & $\begin{array}{l}\text { SPR moves to } \\
\text { Villarreal et al.'s tree }\end{array}$ & $\begin{array}{l}\text { Shared nodes with the } \\
\text { BW molecular tree }\end{array}$ & $\begin{array}{l}\text { Shared nodes with } \\
\text { Villarreal et al.'s tree }\end{array}$ \\
\hline K7 morphological tree & $\mathbf{8 . 1}$ & $\mathbf{7 . 8}$ & 17 & $\mathbf{1 8}$ \\
$\begin{array}{l}\text { Bischler's morphological tree } \\
\text { Boisselier-Dubayle et al.'s } \\
\text { morphological tree }\end{array}$ & 9.1 & 9.2 & 10.7 & 17 \\
\hline
\end{tabular}

BW, block weighting; SPR, subtree pruning and regrafting.

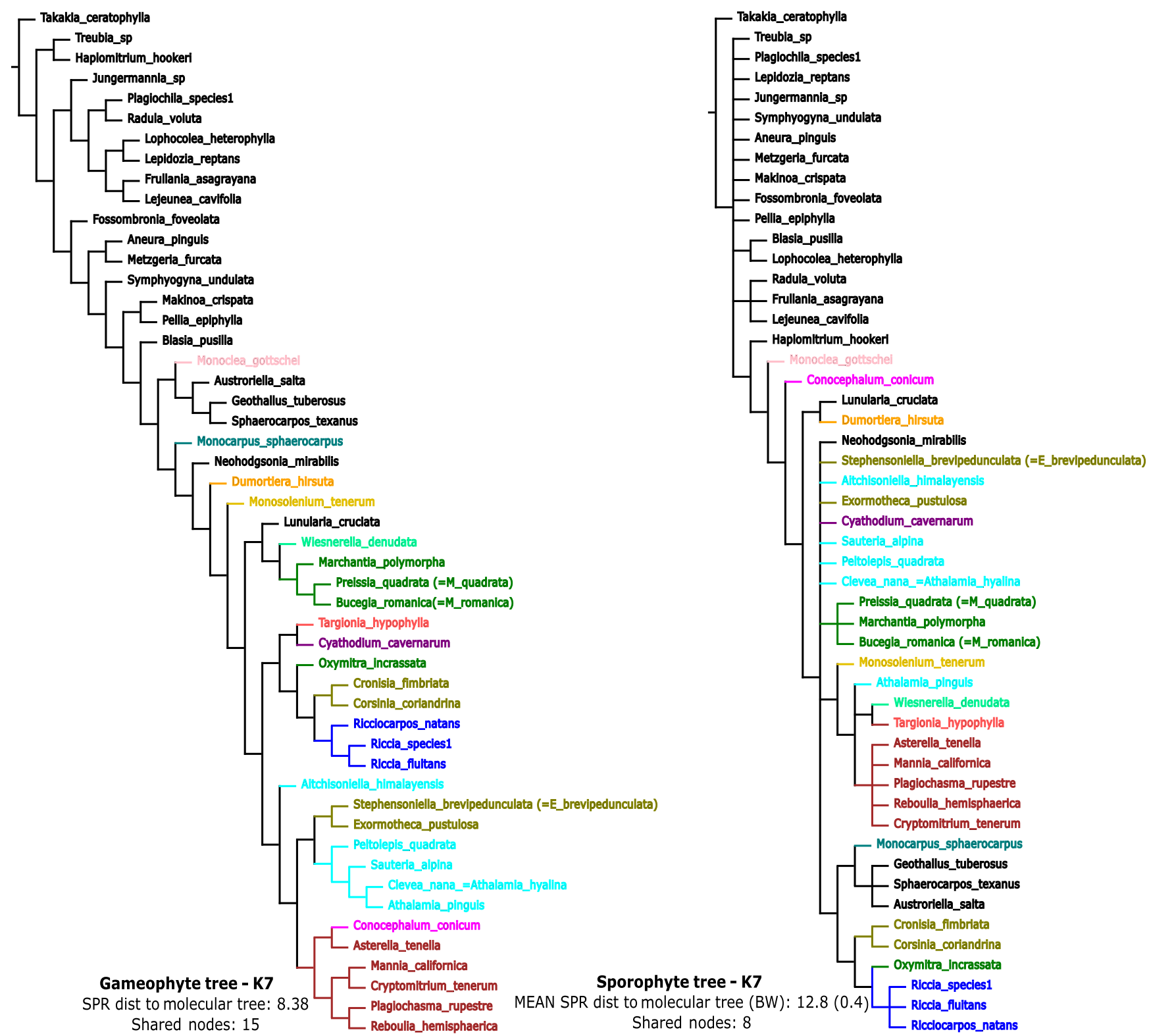

Fig. 4. Morphological trees derived from gametophytic characters and sporophytic characters under implied weighting $(K=7)$. Families within Marchantiales are highlighted. SPR values and shared nodes with the molecular tree are indicated below each tree. For sporophytic tree, the average SPR value is provided (SD in parenthesis). [Colour figure can be viewed at wileyonlinelibrary.com]. 


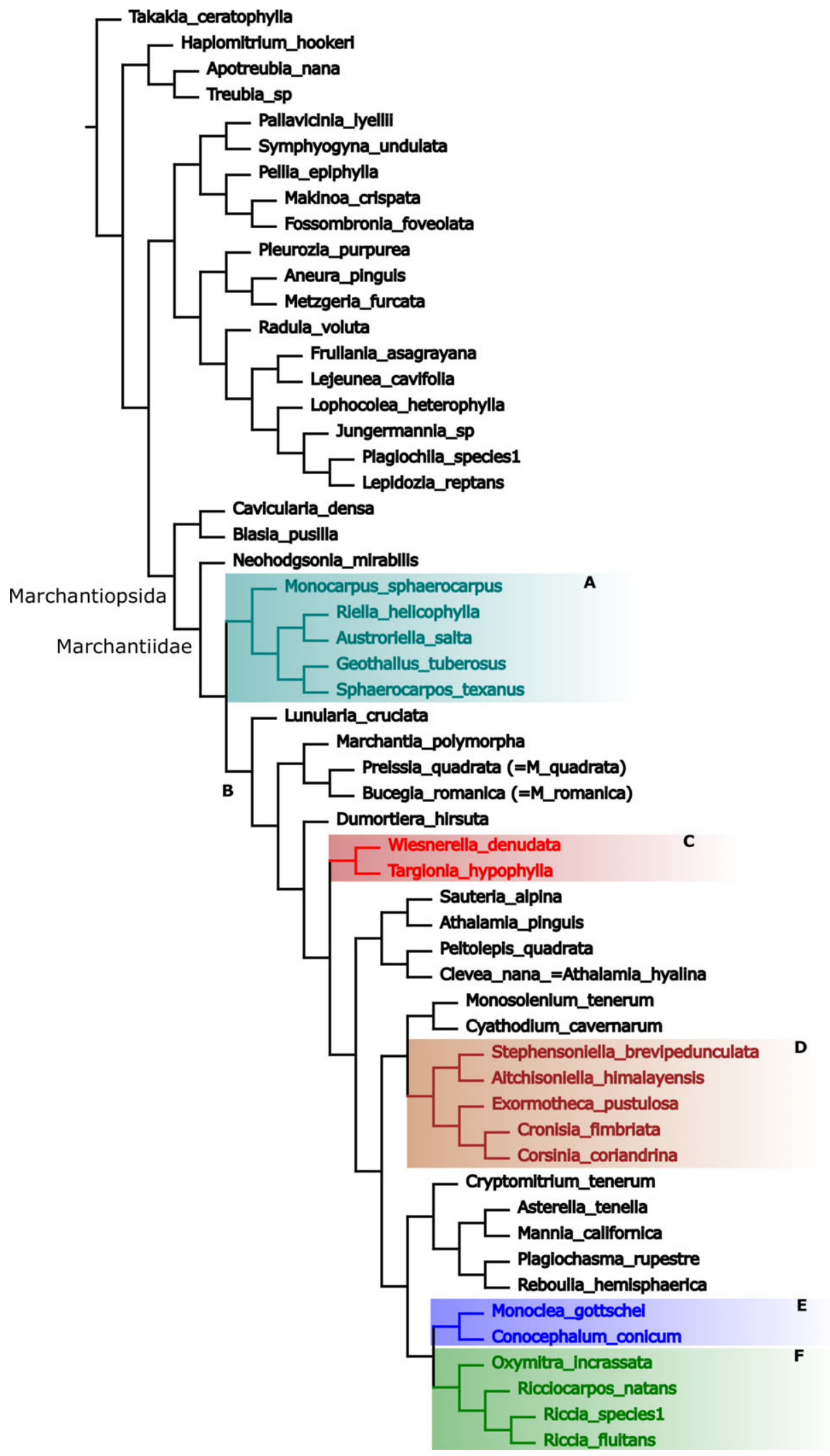

Fig. 5. Groups with improved diagnoses (Combined tree under BW). A detailed diagnosis for each group is in Table 3 . The selected inter-family level nodes were those groups simultaneously well supported and diagnosed. [Colour figure can be viewed at wileyonlinelibrary.com]. 
Table 3

Synapomorphies for mid- and high-level groups within Marchantiidae and Marchantiopsida

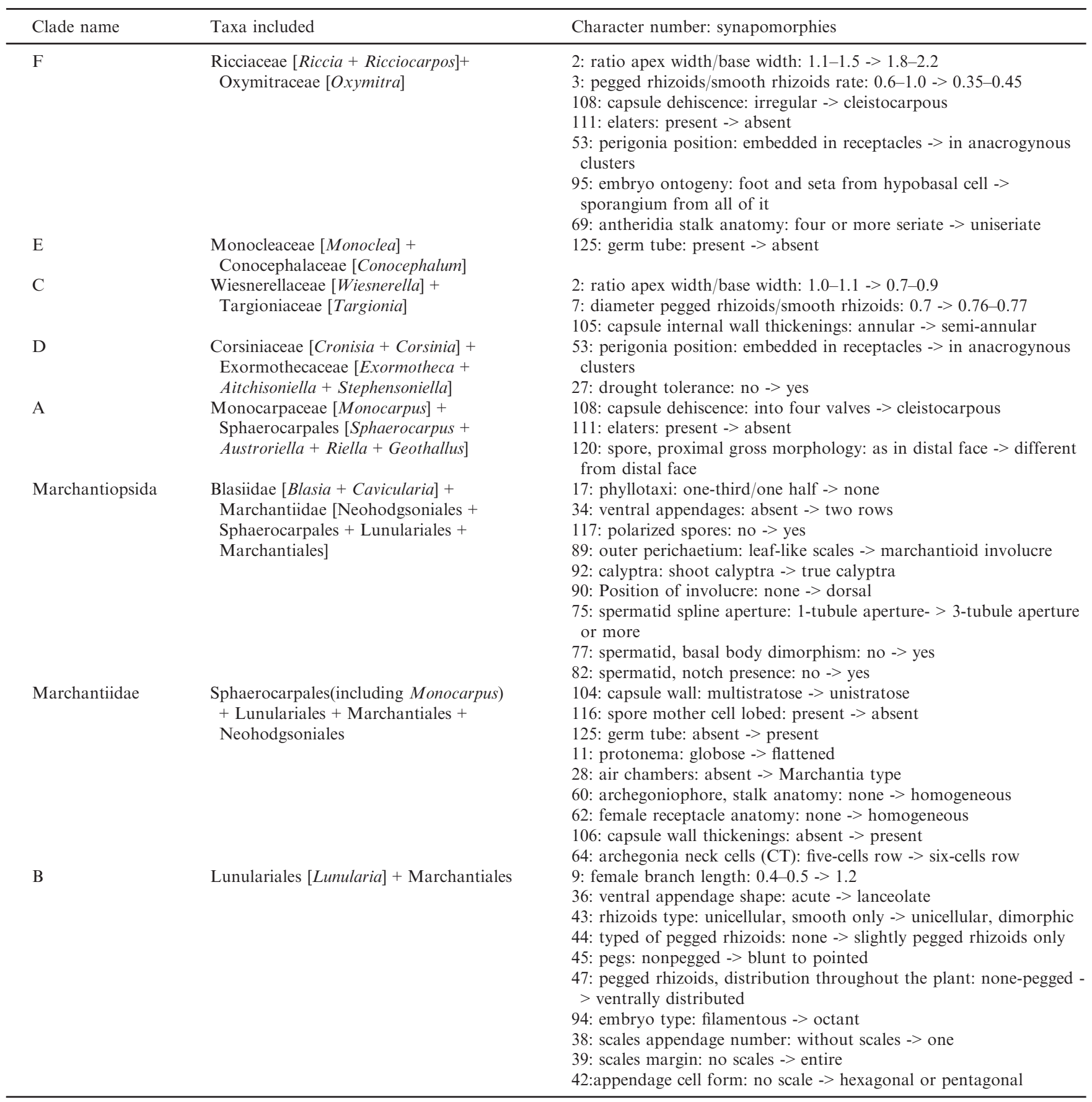

synapomorphies for each node in the phylogeny is in Table S2).

Within the entire phylogeny, continuous characters diagnosed 18 clades (16 within Marchantiopsida). Additionally, 18 clades were diagnosed by at least one sporophytic feature. In ten out of these 18 nodes, $50 \%$ or more of the diagnostic traits were derived from the sporophyte (Fig. 6; Fig. S2).
The distribution of this proportion per taxonomic range was observed to be higher for orders than for classes or subclasses. The inclusion of sporophytic characters into the diagnosis at the interfamily level was also considerably high. In fact, order and interfamily nodes received the highest sporophyte contribution in terms of synapomorphies (Fig. 6; Fig. S2). 


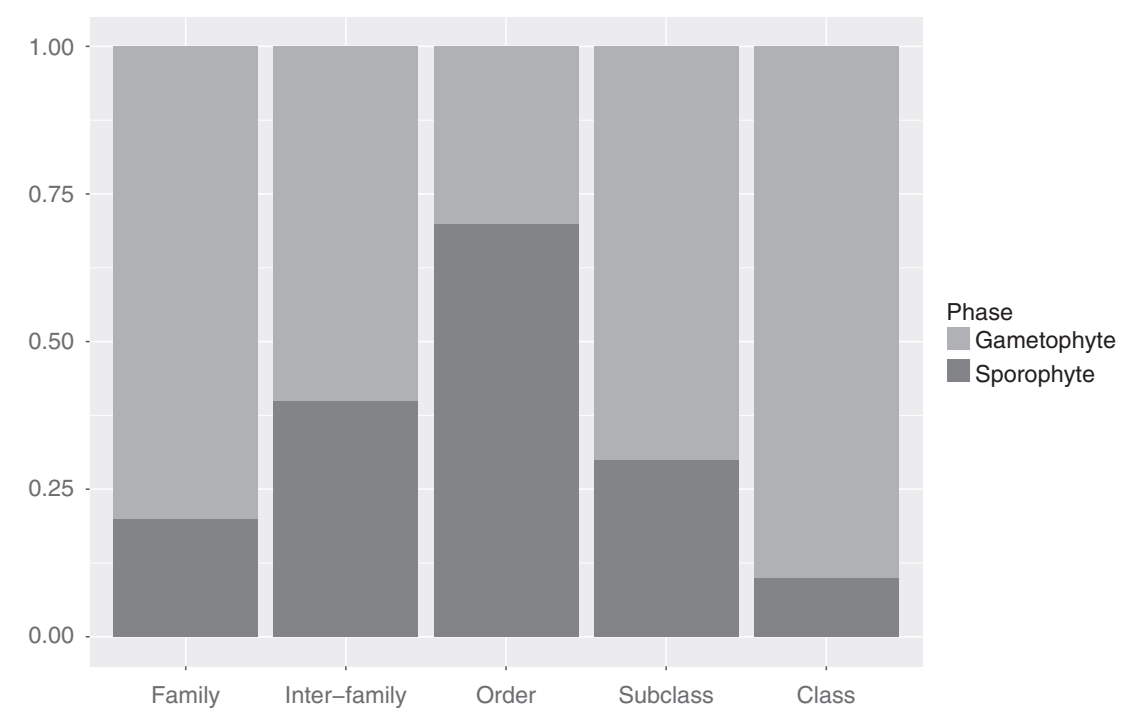

Fig. 6. Percentage of synapomorphies per ontogenetic phase per taxonomic category. $\mathrm{C}=$ class; $\mathrm{SC}=$ subclass; $\mathrm{O}=$ order; $\mathrm{F}=\mathrm{family}$ and $\mathrm{X}=$ inter-family. Proportion of sporophytic synapomorphies mapped along the combined tree is included as Fig. S2 in the Supplementary material.

\section{Mapping life-history traits}

None of the ecological groups defined by Bischler (1998) had a single origin (Fig. 7). Group II was the basal group along Marchantiidae, whereas groups III and IV characterized distant nodes to Sphaerocarpales. Group I was polyphyletic in three different clades (Fig. 7). Capsule dehiscence was reconstructed with 13 steps; the cleistocarpous state diagnosed Sphaerocarpales + Monocarpus, Oxymitraceae + Ricciaceae and Corsinia + Cronisia. The optimization of the number of spores per capsule required nine steps. The continuous characters (spore size and branch length), had 1.5 and 2.15 steps each. Spore size was largest among morphologically simple groups (Oxymitraceae + Ricciaceae + Aytoniaceae + Conocephalaceae + Monocleaceae), whereas smaller sizes were concentrated in the basal clades. Conversely, gametophyte branch length showed lower values in younger clades and higher values among deep nodes. The number of spores per capsule tended to be lower at distant groups and in the basally placed group A (Fig. 7).

\section{Discussion}

In the current work, we present the results of the largest combined analysis for the complex thalloid liverworts. Novel sources of morphological information were evaluated including continuous and structural characters; features used in previous studies were reinterpreted. The results obtained from the combined data corroborate many of the recent proposals but cast doubt on the monophyly of some families. The relative contribution of different types of morphological characters indicate that sporophytic characters provide considerable information for low taxonomic levels. The exhaustive morphological character sampling allows improvement of the analysis in terms of data congruence, sheds light on phylogenetic relationships among families, and improves the diagnoses of high-level groups. The main taxonomic and systematics conclusions presented below are derived from the combined tree under BW. A discussion on group stability and character contribution derives from the results of the partitioned analyses.

\section{Monophyly and stability of groups}

Most of the deep relationships within the subclass Marchantiidae are in agreement with previous studies (Wheeler, 2000; Boisselier-Dubayle et al., 2002; Forrest et al., 2006; Villarreal et al., 2016). The order Marchantiales, as conceived by Crandall-Stotler et al. (2009) or Bischler (1998), is not retrieved as monophyletic in any of our analyses (Fig. 1). After finding a close association between Monocarpus and Sphaerocarpales, Forrest et al. (2015) and Villarreal et al. (2016) rejected Marchantiales sensu Crandall-Stotler et al. (2009), which agrees with our finding of a welldiagnosed Clade A (Monocarpus + Sphaerocarpales; Figs 1 and 5).

The present analyses recovered several interesting groups above the family level (Figs 1 and 5). The earlier removal of the genus Lunularia from Marchantiales led to the proposal of the order Lunulariales (Long, 2006; Crandall-Stotler et al., 2009). Relationships among high-level taxa remained unresolved 


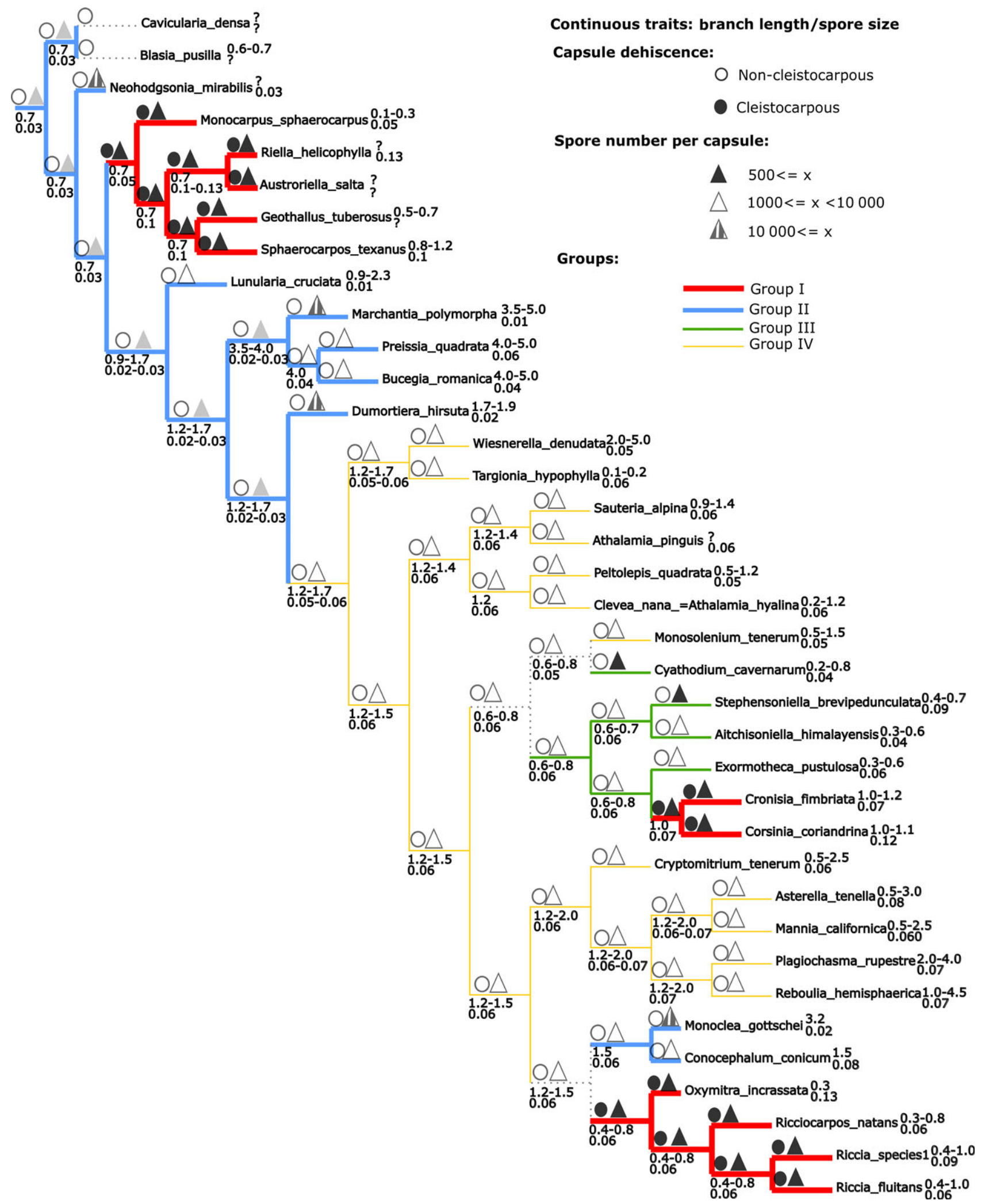

Fig. 7. Character mapping of selected adaptive features across ingroup (Combined tree under BW). Branches coloured according Bischler's lifehistory groups. Selected characters shown as continuous characters below branches [branch length $(\mathrm{cm}) /$ spore size $(\mathrm{mm})]$ and coloured symbols. Grey symbols and dotted branches represents ambiguous optimisations. [Colour figure can be viewed at wileyonlinelibrary.com]. 
throughout different studies (Crandall-Stotler et al., 2009), and thus the proposal of four different orders within Marchantiidae seemed appropriate (Sphaerocarpales, Neohodgsoniales, Lunulariales and Marchantiales). However, Villarreal et al. (2016) found a close association between Lunulariales and Marchantiales (except Monocarpus). Our analyses recover the same group, Clade B (Figs 1 and 5), which differs from others definitions of Marchantiales (Bischler, 1998; Crandall-Stotler and Stotler, 2000; Long, 2006; Crandall-Stotler et al., 2009) in excluding Monocarpus and including Lunularia (as Lunulariales). Although the distinction of two different orders (Lunulariales and Marchantiales) is not necessarily contradicted, it does not seem to be completely suitable given the present results. This is strengthened by the fact that the node comprising Marchantiales (except Monocarpus) lacks diagnostic characters and Lunularia only shows a low number of autapomorphic changes (Fig S1 and Table S2).

Many of the clades recovered at mid-taxonomic levels by Villarreal et al. (2016; i.e. those which grouped two families) are also found in the present study. The Clade F (Riccia + Ricciocarpos + Oxymitra; Fig 5) was formerly considered as an order (CrandallStotler and Stotler, 2000) or suborder (Bischler, 1998). This clade was previously recovered with high support value (Villarreal et al., 2016), and our results agreed in the three analyses (combined, molecular and morphological data; Fig. 1). The rest of the interfamilial groups have not been recognized formally in modern classifications. Clades E (Conocephalum + Monoclea) and C (Wiesnerella + Targionia; Fig. 5) are both highly supported and stable in the combined and molecular data. Clade D (Stephensoniella + Aitchisoniella + Exormotheca + Corsinia + Cronisia $)$ is exclusively recovered by our combined and molecular data (Figs 1 and 5), contradicting recent changes (see below).

The outcomes of the present study show discrepancies with the recently made nomenclatural changes at the family level and below (Long and Crandall-Stotler, 2016; Long et al., 2016a,b). The genus Aitchisoniella was recently transferred to Cleveaceae (Long et al., 2016b) on the basis of its sister relationship with the remaining genera of the family (Villarreal et al., 2016). In contrast to the results of Villarreal et al. (2016), the position of Aitchisoniella within Cleveaceae is not supported by our data (Fig. 1; see Sensitivity plots). In our analyses, on the one hand, Aitchisoniella was resolved as sister to Stephensoniella with high support (SR 100) and clearly nested in Corsiniaceae (Fig. 1; Clade D in Fig. 5). Exormotheca, on the other hand, was recovered as sister to Corsinia and Cronisia in the analyses based on both the combined and molecular data (Fig. 1). Morphology, nonetheless, produces puzzling results regarding Corsiniaceae. As in Villarreal et al. (2016), morphological trees recovered Exormotheca and Stephensoniella as sister taxa but these were unrelated to the remaining Corsiniaceae (Fig. 3). Hence, the nomenclatural changes proposed to both Cleveaceae and the genus Stephensoniella (Long et al., 2016a,b) are not supported by the results of our analyses. Instead, our results suggest that Corsiniaceae should be reviewed to accommodate Aitchisoniella, and that Stephensoniella and Exormotheca are actually independent taxa.

The genera Corsinia and Cronisia were found to be sister taxa by the first time in a combined analysis (Fig. 1). Although this clade was resolved here with a moderate support value, it was found in most of the analytical conditions (13 out of 17); showing a high stability (Fig. 1). The fact that Villarreal et al. (2016) recovered a clade consisting of paraphyletic "traditional" families (Corsiniaceae and Exormothecaceae; fig. 2 in Villarreal et al., 2016), led to a logical rearrangement of these groups (Long et al., 2016a,b). Compared to previous studies (Boisselier-Dubayle et al., 2002; Forrest et al., 2006; Villarreal et al., 2016), the inclusion of extensive morphological data turned over many relationships at the genus level. Unlike Stephensoniella and Exormotheca (Villarreal et al., 2016), the novel link between Cronisia and Corsinia is supported by a large number of morphological characters. In addition, both genera have few autapomorphic characters (Table S2). Altogether, this makes Cronisia and Corsinia suitable taxa for being merged under a single generic name.

As Crandall-Stotler et al. (2009) pointed out, trying to solve the deep relationships inside Marchantiidae has commonly challenged researchers. Our results show Clade B (Marchantiales and Lunulariales; Fig. 5) is an unstable clade in the molecular dataset and absent in the morphological trees (sensitivity plots in Fig. 1). By contrast, the Clade B showed up as a stable group in the combined analysis. These outcomes suggest that adding morphological data to molecular datasets improves the stability of the clade regardless of the overall incongruence between partitions. Additionally, Clade B received a higher support value when morphological data was included. Similarly, support values of other less-inclusive groups (Sphaerocarpales, Ricciaceae and Corsiniaceae) were significantly improved after the addition of morphology (Table S3). These results highlight the fact that extensive morphological data can successfully capture similar patterns to those obtained with molecular data. Even more, unstable or poorly supported results derived from molecular data can be significantly improved after the addition of morphology.

The differences in the results as compared with previous studies may be a consequence of both the addition of new data and the different methods employed 
to derive the phylogenetic hypotheses. It has been pointed out that nodes with low support may be sensitive to changes in analytical conditions (Giribet, 2003; Miller and Hormiga, 2004). Under this reasoning, a clade founded on scarce evidence could be unstable to the addition of new conflicting characters. The differences between our results and previous phylogenies focused mainly on Corsiniaceae and Cleveaceae. In Villarreal et al.'s (2016) phylogeny, the groups Corsiniaceae + Exormotheca and Exormotheca + Stephensoniella had low to moderate support (BS $<50$ and 79 , respectively). In our study, these taxa are highly unstable, especially Exormotheca (see sensitivity plots in Fig. 1). Additionally, recent phylogenies were mostly conducted under maximum-likelihood or Bayesian analyses (Forrest et al., 2015; Villarreal et al., 2016), but implied weighting was never used for analysing data of this group. Thus, the differences in the results might be at least partially attributed to the different analytical methods.

\section{Contribution of data, conflict and agreement}

Boisselier-Dubayle et al. (2002) and Crandall-Stotler et al. (2005) have previously analysed the incongruence in results based on different data types in Marchantiales. Even if exhaustive, such comparisons did not quantify the degree of conflict and contribution of specific groups of morphological characters to recover clades. The topological comparisons (SPR distance and shared nodes) carried out in the present analysis showed the high similarity of the molecular trees with the combined tree. Boisselier-Dubayle et al. (2002) stated that given a large amount of molecular data, such an outcome should be expected. However, the extension to which morphological results depart from molecular trees has been rarely evaluated. In this study, the SPR measures indicated that our morphological trees were similar to the molecular trees (8.1 SPR movements; Fig. 3; Table 2). The comparisons between the molecular trees and the morphological trees of Bischler (1998) and Boisselier-Dubayle et al. (2002) yielded SPR values above 9 (Table 2). In terms of shared nodes, Bischler's tree is similar to our BW molecular tree (Table 2). This similarity was explained by the monophyly of the traditional Exormothecaceae in both Bischler's tree and our BW molecular tree. The alternative comparison, using Villarreal et al.'s (2016) tree as reference, retrieved the K7 morphological tree as the most similar (Table 2). Consequently, our K7 morphological tree maximized similarity with molecular hypotheses in three out of four comparisons.

It has been formerly stressed that morphology-based trees and molecular-based trees rendered markedly different topologies (Boisselier-Dubayle et al., 2002).
Although this conflict is also reflected in our trees (Fig. 3), the K7 morphology-based tree has both dissimilarities with previous morphological trees and common aspects with molecular trees (Bischler, 1998; Crandall-Stotler and Stotler, 2000; Boisselier-Dubayle et al., 2002). As previous authors have pointed out, morphological trees tended to place morphologically complex species in more distant positions regarding Sphaerocarpales (Boisselier-Dubayle et al., 2002; Forrest et al., 2006; Crandall-Stotler et al., 2009). In contrast, our morphological trees somewhat resemble the topology of molecular trees (Fig. 3; see online supplemental material). In the $\mathrm{K} 7$ morphological tree, the morphologically simple clade $\mathrm{F}$ was recovered in a distant position regarding Sphaerocarpales (Fig. 3); contrasting with earlier morphological phylogenies (Boisselier-Dubayle et al., 1997, 2002; Bischler, 1998; Crandall-Stotler and Stotler, 2000). The genera Corsinia and Cronisia were closely related to group F, as in Forrest et al. (2006). The "traditional" Exormothecaceae was rejected (Aitchisoniella + Stephensoniella + Exormotheca; as in Villarreal et al., 2016) and Neohodgsonia was excluded from Marchantiaceae (as in Forrest et al., 2006). Nonetheless, our morphological trees also have some coincidences with former morphological trees. For example, they exclude Monoclea from Marchantiales and do not support Lunulariales (as in Crandall-Stotler and Stotler, 2000). In this sense, results from our morphological data can be interpreted as intermediate between the equallyweighted morphological trees of previous studies (Bischler, 1998; Crandall-Stotler and Stotler, 2000; Boisselier-Dubayle et al., 2002) and various molecular hypotheses (Boisselier-Dubayle et al., 2002; Forrest et al., 2006; Villarreal et al., 2016; and this study).

The most in-depth analysis to elucidate the extension of data conflict between sets of morphological characters was performed by Crandall-Stotler et al. (2005). They concluded that sporophytic characters are not more informative than gametophytic characters. In our study, the topological similarity with the molecular tree is maximized by the tree derived from the gametophytic characters (Fig. 4). Nevertheless, this does not imply that sporophytic characters are uninformative. Although some groups are in conflict compared with trees derived from other kind of characters (e.g. Sphaerocarpales' nested position into Marchantiales), several taxonomic groups in the molecular tree are also found in the sporophyte tree but not in the gametophyte tree (Fig. 4). Clade F was found in the combined analysis and also in the sporophytic tree, in agreement with previous phylogenies (Bischler, 1998; Crandall-Stotler and Stotler, 2000; Wheeler, 2000; Boisselier-Dubayle et al., 2002; Forrest et al., 2006; Villarreal et al., 2016; Fig. 4). Similarly, the family Aytoniaceae is supported by the sporophytic data but 
rejected by gametophytic characters (Fig. 4). Sporophytic traits also resolved two additional clades in accordance with molecular hypotheses (Fig. 5): groups C (Wiesnerella and Targionia) and A (Monocarpus and Sphaerocarpales; Wheeler, 2000; Boisselier-Dubayle et al., 2002; Forrest et al., 2006; Villarreal et al., 2016).

Our data show that trees based on morphological data have a certain level of agreement with the molecular topology in terms of SPR distance, shared nodes and groups placement. The increased similarity compared to previous morphological datasets is probably related to the inclusion of new information and the reinterpretation of previous characters. Until now, morphological datasets were relatively small (43 discrete characters) and none of the larger matrices was concentrated on Marchantiidae (Crandall-Stotler and Stotler, 2000; He-Nygrén et al., 2006). In addition, it is worth noting that Boisselier-Dubayle et al. (1997) previously suggested using weighting approaches for diminishing the effect of incongruence between data types. Homoplasy reported by earlier works (Boisselier-Dubayle et al., 2002; Crandall-Stotler et al., 2005) was now downweighted by the use of implied weighting (Goloboff, 1993). Altogether, these factors can be considered as improvements in the analysis of morphological data.

\section{Synapomorphies and Diagnosis}

Crandall-Stotler et al. (2009) remarked on the fact that some characters could not be confidently assigned to several groups within the current classification. Many of the groups diagnosed for the first time in the present study were already recovered in previous analyses (Forrest et al., 2006; Villarreal et al., 2016), yet they could not be fully evaluated on the basis of morphological characters. Consequently, their morphological definition remained dubious. In the present combined study, the diagnosis of several groups was clarified.

In the contemporary classification (Crandall-Stotler et al., 2009), Marchantiopsida and Marchantiidae were described in general terms. That is, some characters were actually apomorphic (e.g. cuneate apical cell or uni-stratose capsule wall; Crandall-Stotler et al., 2005) whereas others were non-apomorphic traits (e.g. plants thalloid, rarely leafy or dehiscence by valves, lid or cleistocarpous). In our BW combined tree, these groups are supported by nine morphological characters each, mainly gametophytic features (Fig. 6; Table 3; Fig. S2). Clade B (Marchantiales and Lunulariales; Fig. 5) has not been diagnosed since its original recovery (Wheeler, 2000; Boisselier-Dubayle et al., 2002; Forrest et al., 2006). In the present combined analysis, such a node is delimited by 15 synapomorphies, most of these scored from the gametophytic phase.
Hexagonal/pentagonal scale cells, lanceolate shaped scales and thin pegged rhizoids are examples of diagnostic characters for this group.

Most of the previous analyses (Wheeler, 1998, 2000; Forrest et al., 2006; He-Nygrén et al., 2006; Villarreal et al., 2016) recovered Clade F (Oxymitra, Ricciocarpos and Riccia; Fig. 5). However, it was not recognized within the contemporary classification (Crandall-Stotler et al., 2009). This group was defined almost exclusively by sporophytic characters in previous morphological analyses (Bischler, 1998; Boisselier-Dubayle et al., 2002). In our study, this node is diagnosed by seven morphological synapomorphies, four of them being gametophytic traits. Thus, the number of synapomorphies not only increased but also new gametophyte-related characters are added. A distinctive diagnostic trait is the ratio between apex and base width of 1.8-2.2; indicating an obcordate thallus shape. A $0.3-0.4$ proportion of pegged rhizoids/smooth rhizoids is likewise diagnostic for this clade. Antheridia (male gametangia) primarily positioned in anacrogynous clusters (not derived from an apical cell; as opposed to being gathered in receptacles or specialized branches) is a further gametophytic synapomorphy of this node.

Molecular evidence has consistently recovered both Clades E (Monoclea and Conocephalum; Forrest et al., 2015; Villarreal et al., 2016) and C (Wiesnerella and Targionia; Boisselier-Dubayle et al., 2002; Forrest et al., 2006; Villarreal et al., 2016); but none of these studies could provide synapomorphic morphological characters diagnosing both clades, as found here. Clade $\mathrm{E}$ is diagnosed by the absence of a germinal tube, whereas $\mathrm{C}$ is defined by three synapomorphies: a ratio of $0.7-0.9$ between apex and base width, a 0.76 0.77 proportion of pegged rhizoids and semi-annular capsule thickenings (as opposed to annular thickenings in remaining clades).

The results obtained in the present analysis share many nodes with previous phylogenetic hypotheses (Forrest et al., 2006, 2015; Villarreal et al., 2016) although the diagnoses of some nodes differ from that previously proposed. Many characters were potentially linked to the recently proposed Corsiniaceae (Exormotheca + Corsinia + Cronisia; Long et al., 2016a). However, only two characters are synapomorphies of this clade: pegged rhizoids originating near the thallus apex and a multiple-of- 8 chromosome number. No spore-related character supported the link between Exormotheca and Cronisia + Corsinia as suggested (Long et al., 2016a). The novel Clade D, which includes Aitchisoniella and Corsiniaceae (Exormotheca, Stephensoniella, Cronisia and Corsinia), was diagnosed by two vegetative traits: antheridia in anacrogynous clusters and drought tolerance. Nevertheless, it must be noted that the diagnosis of Clade D is not comparable to the new Corsiniaceae (Long et al., 2016a) 
because it comprises different taxa (i.e., includes Aitchisoniella). Clade A (Monocarpus and Sphaerocarpales; Fig. 5) was recently found by Forrest et al. (2015) who proposed that this group could be described on the basis of sporophytic trait reductions. Our results confirmed Forrest et al.'s proposal by mapping reversions as synapomorphic character changes (cleistocarpous capsules, no elaters and distinctive ornamentation of the spore proximal face).

Diagnoses of the remaining families with more than one genus were also modified relative to Crandall-Stotler et al.'s (2009) classification, as a result off Aytoniaceae and Cleveaceae (excluding Aitchisoniella) constraining their original delimitations. Nevertheless, 10 and eight synapomorphies are found for each taxon, respectively. Many of these apomorphies were already proposed by Crandall-Stotler et al. (2009) whereas others are completely new characters. For instance, a 1.09-1.1 pegged rhizoids/smooth rhizoids proportion and simple pegged rhizoids distributed in stalk furrows are new synapomorphies for Aytoniaceae. Cleveaceae (excluding Aitchisoniella) is fairly well diagnosed by a high peg density (9.411.0), mid to long pegs (4.5-4.6) and tuber presence, among others. Marchantiaceae rendered as apomorphies many of the characters previously proposed by CrandallStotler et al. (2009). However, novel characters related to pegged rhizoids are also added. Ricciaceae, as was the case in several classifications (Bischler, 1998; CrandallStotler et al., 2009), is circumscribed mainly by reversals (absences).

Two additional features characterize the new diagnoses presented in this study: a high proportion of sporophytic characters and input of continuous characters. Bischler (1998) documented an important number of synapomorphies related to sporophytic characters. Namely, $52 \%$ of her sampled sporophytic characters presented apomorphic states (Bischler, 1998). That percentage was informative neither on the ubiquity of changes nor the proportion of sporophytic apomorphies per node. After a scrutiny of the synapomorphies reported by Bischler (1998), it turned out that the sporophytic phase contributed 46 out of 126 changes ( $36 \%$; mainly concentrated at deep nodes). In our analysis, synapomorphies are dominated by gametophytic features at the family level (Fig. 6; Fig. S2). These quantities may coincide with the notion that sporophytic traits provide scarce information at low taxonomic levels (Schuster, 1984a,b; Crandall-Stotler and Stotler, 2000). However, this idea is not supported when the synapomorphies presented at the level of orders and nodes that grouped families are considered. These nodes were diagnosed by a large number of sporophytic traits (Fig. 6; Fig. S2). Conversely, sporophytic features are poorly represented in the diagnosis at the level of subclasses and classes. The relatively high number of sporophytic synapomorphies at low taxonomic levels compared to that obtained in
Bischler (1998) could be related to the higher number of sporophytic characters included in our dataset. Indeed, Bischler's (1998) dataset included 12 sporophytic characters whereas our matrix scored 34 sporophytic traits. These new findings provide a basis upon which new groups could be proposed relying on both high support values and a clearly stated diagnosis.

Several synapomorphies retrieved in the present analysis involve continuous characters, a novel result given that previous phylogenetic analyses in this group did not include this type of character. The lack of continuous characters in earlier studies (Bischler, 1998; Boisselier-Dubayle et al., 2002) can be explained by the absence of a method that could deal with continuous variation, because Goloboff et al. published their approach later, in 2006. However, recent studies have explicitly suggested that continuous characters be excluded. Oyston et al. (2016) argued that at higher taxonomic categories $(\approx$ deeper nodes), taxa tend to differ more contrastingly than at lower levels $(\approx$ shallow nodes). Consequently, Oyston et al. claimed that classical morphometrics (continuous characters) are not suitable for deep levels. Discretized characters were considered better because they exhibit a gap in the continuous trait being evaluated (Oyston et al., 2016). The analysis of continuous characters analysed as such (Goloboff et al., 2006) led us to diagnose 16 out of 35 ingroup nodes using this type of data. Indeed, even if the consensus is poorly resolved, some nodes at the family level and at middle level are recovered when continuous characters are used to infer phylogenetic relationships (Fig. S4). Thus, our findings contradict the notion that continuous traits are appropriate only for shallow taxonomic levels. Further surveys on continuous characters should be conducted in order to completely elucidate their relevance in liverwort phylogeny.

\section{Life history traits, morphological resemblance and evolutionary scenarios}

Although some morphological traits were slightly decoupled regarding life-history groups, a general correlated pattern is clearly recovered (Fig. 7). Bischler (1998) established such life-history groups on the grounds of a statistical analysis of environmental factors and morphological features. By mapping these groups onto her morphological tree, she suggested that morphology and life strategies co-variation is phylogenetically structured. ${ }^{1} \quad$ Therefore, morphological

\footnotetext{
'Note that Bischler (1998) changed group labelling in her phylogenetic mapping (fig. 15 in Bischler, 1998). However, the groups remained the same [e.g. Marchantiaceae and Conocephalaceae were first placed in group 2 (Bischler, 1998, p. 135) and then in group 4 (p. 136)].
} 
resemblance was argued to be caused by ancestry rather than by environmental pressure. The character reconstruction in our combined tree is in partial agreement with Bischler's hypothesis. Explaining the morphological diversity in terms of common ancestry would require each life-history group to be monophyletic or paraphyletic. Because groups II-IV had a unique origin, morphological resemblance within each group could be attributed to common ancestry. Similarly, within each clade of Group I taxa tended to be highly similar with regard to morphological characters (Fig. 7). Environmental pressure could be considered to explain morphological diversity among unrelated taxa. Unlike members of the same clade, similarity between unrelated taxa could not be caused by common ancestry. The resemblance between, say, the genera Riccia and Monocarpus is easier to explain in terms of similar environmental pressure (Fig. 7). Our findings, therefore, allow Bischler's hypothesis to be reformulated. That is, based on the current phylogenetic patterns, morphological resemblance is partially explained by common ancestry (inside each clade) and partially by convergence (between unrelated members of the group I).

Multiple independent character reductions occurred in taxa of Group I, commonly limited to open dry habitats (cleistocarpous capsules, small gametophytes, few and large spores). These features are favoured under environmental stress (Bischler, 1998; Bischler et al., 2005). Conversely, members of groups II-IV tended to develop morphologically complex features (larger gametophytes, noncleistocarpous capsules, numerous and small spores), appropriate for mesic habitats (Bischler, 1998). Thus, the correlation between morphological traits and life strategies found by Bischler (1998) is confirmed.

Deviations from the expected evolutionary trends (Bischler, 1998) were a consequence of both dissimilar topologies and methodological issues. The statistical survey of Bischler (1998) was conducted without considering phylogeny as a source of variation constraint. Rather, species were treated as independent statistical entities. In order to evaluate the concerted evolution of potentially adaptive characters, the effect of the phylogeny should be eliminated. By doing so, species could be treated as statistically independent units (Felsenstein, 1985). An exhaustive quantitative evaluation of putative adaptive characters is still needed. At the moment, the adaptive value of morphology remains a poorly investigated area.

\section{Final remarks}

The first in-depth combined analysis for the complex thalloid liverworts was conducted. An improved character sampling led to the construction of the largest morphological dataset. Key findings were achieved regarding morphological contribution and diagnosis improvement. In sum, the well-established assumption that morphology may produce completely incongruent patterns with molecular data was discredited. Indeed, many nodes recovered with low support values in previous studies were retrieved by our combined data with increased support. This suggests that the combination of apparently conflicting data types may reveal hidden support for most of the groups.

It is argued that common weaknesses of morphological data are challenging character circumscription and, for this specific group, no informative changes (Boisselier-Dubayle et al., 2002). Morphological data are often described as highly homoplastic in plants (Buck et al., 2000; Ranker et al., 2004; Liu et al., 2012; Yu et al., 2013; Wu et al., 2015; among others). Such a characterization became a widespread notion for different groups of organisms. Scotland et al. (2003) even suggested that, regardless of the taxonomic group, morphological characters should not be evaluated given their problematic nature. Our results, as well as others' (e.g. Wahlberg et al., 2005), strongly reject such a statement. The inclusion of more and novel characters produced topologies which differ from small-matrix-derived morphological phylogenies and are more similar to molecular trees. Likewise, it was shown not only that the number of diagnosed groups increased, but also that most of the previously proposed diagnoses were imprecise.

Several synapomorphies at the interfamily level were found for the first time and some unsupported taxonomic changes were questioned. Although deep relationships among derived families are still dubious, diagnoses and support values of many interfamily nodes were improved. Subsequently, this could be translated into new groups gathering derived families. The taxonomy of the families Cleveaceae and Corsiniaceae, as well as the genera Aitchisoniella and Stephensoniella, shall be reviewed. At the present, finding a proper set of synapomorphies for the order Marchantiales is still a major problem. A reasonable approach would be to redefine Marchantiales so as to include its sister taxon Lunulariales. By doing this, a supported and accurately diagnosed category could be proposed. The fact that morphology-derived trees improved their congruence with molecular evidence (increased values of shared nodes and SPR distance) will encourage the survey of more and new morphological characters.

\section{Acknowledgments}

We thank Emilio Cano (Real Jardín Botánico) for his technical assistance in extracting and amplifying 
plant material. We are also indebted to the curators of the PC herbarium for providing invaluable specimens. Several colleagues also contributed fresh material collected in northern Argentina. J.R.F. is supported by a Doctoral Fellowship of the National Scientific and Technical Research Council (CONICET). This study was funded by FONCyT (PICT-1838, PICT-1930 and PICT -0810), the National University of Tucuman (PIUNT-G524) and CONICET (PUE 0070). TNT is freely available thanks to the Willi Hennig Society.

\section{References}

Beck, R., Lee, M., 2014. Ancient dates or accelerated rates? Morphological clocks and the antiquity of placental mammals. Proc. Biol. Sci. 281, 20141278.

Bischler, H., 1998. Systematics and evolution of the genera of the Marchantiales. Bryophyt. Bibl. 51, 1-201.

Bischler, H., Jovet-Ast, S., 1981. The biological significance of morphological characters in Marchantiales (Hepaticae). Bryologist 84, 208-215.

Bischler, H., Boisselier-Dubayle, M., Pant, G., 1994. On Aitchisoniella Kash (Marchantiales). Cryptogam. Bryol. 15, 103110.

Bischler, H., Gradstein, S., Jovet-Ast, S., Long, D., Salazar Allen, N., 2005. Marchantiidae. Flora Neotrop. 97, 1-262.

Boisselier-Dubayle, M., Lambourdiére, J., Leclerc, M., Bischler, H., 1997. Relations phylogénétiques chez les Marchantiales (Hepaticae). Incongruence apparente entre données morphologiques et moléculaires. C. R. Acad. Sci. 320, 1013-1020.

Boisselier-Dubayle, M., Lambourdière, J., Bischler, H., 2002. Molecular phylogenies support multiple morphological reductions in the liverwort subclass Marchantiidae (Bryophyta). Mol. Phylogenet. Evol. 24, 66-77.

Borovichev, E.A., Konstantinova, N.A., Andrejeva, E.N., 2012. The genus Sauteria nees (Cleveaceae, Marchantiophyta) in Russia. Arctoa 21, 181-188.

Buck, W., Goffinet, B., Shaw, A., 2000. Testing morphological concepts of orders of pleurocarpous mosses (Bryophyta) using phylogenetic reconstructions based on TRNL-TRNF and RPS4 sequences. Mol. Phylogenet. Evol. 16, 180-198.

Campbell, D., 1896. A new Californian liverwort. Bot. Gaz. 21, 9-13.

Cargill, D., Milne, J., 2013. A new terrestrial genus and species within the aquatic liverwort family Riellaceae (Sphaerocarpales) from Australia. Polish Bot. J. 58, 71-80.

Carrizo, L., Catalano, S., 2015. First phylogenetic analysis of the tribe Phyllotini (Rodentia: Sigmodontinae) combining morphological and molecular data. Cladistics 31, 1-28.

Crandall-Stotler, B., 1980. Morphogenetic designs and a theory of bryophyte origins and divergence. Bioscience 30, 580-585.

Crandall-Stotler, B., Stotler, R., 2000. Morphology and classification of the Marchantiophyta. In: Shaw, A., Goffinet, B. (Eds.), Bryophyte Biology. Cambridge University Press, Cambridge, pp. 21-70.

Crandall-Stotler, B., Forrest, L., Stotler, R., 2005. Evolutionary trends in the simple thalloid liverworts (Marchantiophyta, Jungermanniopsida subclass Metzgeriidae). Taxon 54, 299-316.

Crandall-Stotler, B., Stotler, R., Long, D., 2009. Phylogeny and classification of the Marchantiophyta. Edinb. J. Bot. 66, 155.

Duckett, J.G., Ligrone, R., Renzaglia, K.S., Pressel, S., 2014. Pegged and smooth rhizoids in complex thalloid liverworts (Marchantiopsida): structure, function and evolution. Bot. J. Linn. Soc. 174, 68-92.

Duckett, J., Renzaglia, K., 1993. The reproductive biology of the liverwort Blasia pusilla L. J. Bryol. 17, 541-552.

Evans, A., 1920. The North American Species of Asterella. Government Printing Office, Washington, DC, USA.
Farris, J., 1990. Phenetics in camouflage. Cladistics 6, 91-100.

Felsenstein, J., 1985. Phylogenies and the comparative method. Am. Nat. 125, 1-15.

Forrest, L., Crandall-Stotler, B., 2004. A phylogeny of the simple thalloid liverworts (Jungermanniopsida, Metzgeriidae) as inferred from five chloroplast genes. Monogr. Syst. Bot. Mo. Bot. Gard. 98, 119-140.

Forrest, L., Davis, E., Long, D., Crandall-Stotler, B., 2006. Unravelling the evolutionary history of the liverworts (Marchantiophyta): multiple taxa, genomes and analyses. Bryologist 109, 303-334.

Forrest, L., Long, D., Cargill, D., Hart, M., Milne, J., Schill, D., Rodney, D., Villarreal, J., 2015. On Monocarpus (Monocarpaceae, Marchantiopsida), an isolated salt-pan complex thalloid liverwort. Aust. Syst. 28, 137-144.

Giribet, G., 2003. Stability in phylogenetic formulations and its relationship to nodal support. Syst. Biol. 52, 554-564.

Goloboff, P., 1993. Estimating character weights during tree search. Cladistics 9, 83-91.

Goloboff, P., 1999. Analyzing large data sets in reasonable times: solutions for composite optima. Cladistics 15, 415-428.

Goloboff, P., 2008. Calculating SPR distances between trees. Cladistics 22, 589-601.

Goloboff, P., 2014. Extended implied weighting. Cladistics 30, 260272.

Goloboff, P.A., Catalano, S.A., 2012. GB-to-TNT: facilitating creation of matrices from GenBank and diagnosis of results in TNT. Cladistics 28, 503-513.

Goloboff, P.A., Farris, J.S., Källersjö, M., Oxelman, B., Szumik, C.A., 2003. Improvements to resampling measures of group support. Cladistics 19, 324-332.

Goloboff, P., Catalano, S., 2016. TNT version 1.5, including a full implementation of phylogenetic morphometrics. Cladistics 32, 221-238.

Goloboff, P., Pol, D.,. 2005. Parsimony and Bayesion phylogenetics. In: Albert, V. (Ed.), Parsimony, Phylogeny, and Genomics. Oxford University Press, New York, pp. 148-217.

Goloboff, P., Mattoni, C., Quinteros, A., 2006. Continuous characters analyzed as such. Cladistics 22, 589-601.

Goloboff, P., Carpenter, J., Arias, J., Esquivel, D., 2008a. Weighting against homoplasy improves phylogenetic analysis of morphological data sets. Cladistics 24, 758-773.

Goloboff, P., Farris, J., Nixon, K., 2008b. TNT, a free program for phylogenetic analysis. Cladistics 24, 774-786.

Goloboff, P.A., Torres, A., Arias, J.S., 2017. Weighted parsimony outperforms other methods of phylogenetic inference under models appropriate for morphology. Cladistics. https://doi.org/ $10.1111 /$ cla. 12205

Grubb, P., 1970. Observations on the structure and biology of Haplomitrium and Takakia, hepatics with roots. New Phytol. 69, 303-326.

Gupta, A., Udar, R., 1986. Palyno-taxonomy of selected Indian liverworts. Bryophyt. Bibl. 29, 1-202.

Hassel de Menéndez, G., 1963. Estudio de las Anthocerotales y Marchantiales de la Argentina. Opera Lilloana 7, 1-297.

Hattori, S., Sharp, A., Mizutani, M., Iwatsuki, Z., 1966. The systematic position and distribution of Treubia nana. Bryologist $69,488-492$.

Haupt, A., 1920. Life History of Fossombronia cristula. Bot. Gaz. 69, 318-331.

Haynes, C., 1910. Sphaerocarpus hians sp. nov., with a revision of the genus and illustrations of the species. Torrey Bot. Soc. 37, 215-230.

Hedderson, T., Longton, R., 1996. Life history variation in mosses: water relations, size and phylogeny. Oikos 77, 31-43.

He-Nygrén, X., Juslen, A., Ahonen, I., Glenny, D., Piippo, S., 2006. Illuminating the evolutionary history of liverworts (Marchantiophyta) - towards a natural classification. Cladistics $22,1-31$.

Jovet-Ast, S., 1986. Les Riccia de la région Méditerranéenne. Cryptogam. Bryol. 7, 287-431. 
Karol, K.G., McCourt, R.M., Cimino, M.T., Delwiche, C.F., 2001. The closest living relatives of land plants. Science 294, 2351-2353.

Kashyap, S., 1914a. Morphological and biological notes on new and little known west-Himalayan liverworts. I. New Phytol. 13, 206226.

Kashyap, S., 1914b. Morphological and biological notes on new and little-known west-Himalayan liverworts. II. New Phytol. 13, 308323.

Kashyap, S., 1915. Morphological and biological notes on new and little kwon west-Himalayan liverworts. III. New Phytol. 14, 1-18.

Katoh, K., Toh, H., 2008. Recent developments in the MAFFT multiple sequence alignment program. Brief. Bioinform. 9, 286-298.

Kuwahara, Y., 1986. The Metzgeriaceae of the Neotropics. Cramer. Bryophyt. Bibl. 28, 1-254.

Little, E., 1936. The liverworts of Oklahoma. Bryologist 39, 25-34.

Liu, Y., Budke, J.M., Goffinet, B., 2012. Phylogenetic inference rejects sporophyte based classification of the Funariaceae (Bryophyta): rapid radiation suggests rampant homoplasy in sporophyte evolution. Mol. Phylogenet. Evol. 62, 130-145.

Long, D., 2006. New higher taxa of complex thalloid liverworts (Marchantiophyta-Marchantiopsida). Edinb. J. Bot. 63, 257-262.

Long, D., Crandall-Stotler, B., 2016. Taxonomic changes in Cleveaceae (Marchantiidae, Marchantiophyta) - a correction. Phytotaxa 273, 299-300.

Long, D., Forrest, L., Villarreal, J., Crandall-Stotler, B., 2016a. Taxonomic changes in Marchantiaceae, Corsiniaceae and Cleveaceae (Marchantiidae, Marchantiophyta). Phytotaxa 252, $77-80$.

Long, D., Forrest, L., Villarreal, J., Crandall-Stotler, B., 2016b. The genus Aitchisoniella Kashyap (Marchantiopsida, Cleveaceae) new to China, and its taxonomic placement. J. Bryol. 38, 308-311.

Longton, R., 1988a. Adaptations and strategies of polar bryophytes. Bot. J. Linn. Soc. 98, 253-268.

Longton, R., 1988b. Life-history strategies among bryophytes of arid regions. J. Hattori Bot. Lab. 64, 15-28.

Miller, J., Hormiga, G., 2004. Clade stability and the addition of data: A case study from erigonine spiders (Araneae: Linyphiidae, Erigoninae). Cladistics 20, 385-442.

Mirande, J., 2017. Combined phylogeny of ray-finned fishes (Actinopterygii) and the use of morphological characters in largescale analyses. Cladistics 33, 333-350.

Montagne, J., 1852. Note sur le genre Riella et description d'une espèce nouvelle R. reuteri. Ann. Sci. Nat. Bot. 3, 11-13.

Nickrent, D.L., Parkinson, C.L., Palmer, J.D., Duff, R.J., 2000. Multigene phylogeny of land plants with special reference to bryophytes and the earliest land plants. Mol. Biol. Evol. 17, 1885-1895.

Nixon, K., 1999. The parsimony ratchet, a new method for rapid parsimony analysis. Cladistics $15,407-414$.

Oyston, J., Hughes, M., Gerber, S., Wills, M., 2016. Why should we investigate the morphological disparity of plant clades? Ann. Bot. 117, 859-879.

Qiu, Y.L., Cho, Y., Cox, J.C., Palmer, J.D., 1998. The gain of three mitochondrial introns identifies liverworts as the earliest land plants. Nature 394, 671-674.

Ranker, T.A., Smith, A.R., Parris, B.S., Geiger, J.M., Haufler, C.H., Straub, S.C., Schneider, H., 2004. Phylogeny and evolution of grammitid ferns (Grammitidaceae): a case of rampant morphological homoplasy. Taxon 53, 415-415.

Renzaglia, K.S., 1982. A comparative developmental investigation of the gametophyte generation in the Metzgeriales (Hepatophyta). Bryophyt. Bibl. 24, 1-253.

Renzaglia, K., Mcfarland, K., Smith, D., 1997. Anatomy and ultrastructure of the sporophyte of Takakia ceratophylla (Bryophyta). Am. J. Bot. 84, 1337-1350.

Rubasinghe, S.C.K., 2011. Phylogeny and taxonomy of the complex thalloid liverwort family Cleveaceae Cavers. DPhil thesis, University of Edinburgh, Edinburgh, UK.

Schuster, R., 1969. The Hepaticae and Anthocerotae of North America, East of the Hundredth Meridian. Vol 2. Columbia University Press, New York.
Schuster, R., 1980. The Hepaticae and Anthocerotae of North America, East of the Hundredth Meridian. Vol 4. Columbia University Press, New York.

Schuster, R., 1984a. Comparative anatomy and morphology of the Hepaticae. In: Schuster, R. (Ed.), New Manual of Bryology 2. Hattori Botanical Laboratory, Nichinan, Japan, pp. 760-891.

Schuster, R., 1984b. The Hepaticae and Anthocerotae of North America, East of the Hundredth Meridian. Vol 5. Field Museum of Natural History, Chicago.

Schuster, R., 1992. The oil-bodies of the Hepaticae. I. Introduction. J. Hattori Bot. Lab. 72, 151-162.

Schuster, R., Scott, G., 1969. Study of the family Treubiaceae (Hepaticae; Metzgeriales). J. Hattori Bot. Lab. 32, 219-257.

Scotland, R., Olmstead, R., Bennett, J., 2003. Phylogeny reconstruction: the role of morphology. Syst. Biol. 52, 539-548.

Sharp, A., 1939. Taxonomic and ecological studies of eastern Tennessee bryophytes. Am. Midl. Nat., 26, 7-354.

Singh, S., 2014. An appraisal of genus Riccia in India with a note on diversity and distribution of species. Int. J. Sustain. Water Environ. Syst. 6, 35-43.

Sokhi, J., Mehra, P., 1973. Comparative embryology of Athalamia pinguis Falc. and A. pusilla (St.) Kash. J. Hattori Bot. Lab. 37, 1-54.

Steiper, M.E., Seiffert, E.R.,. 2012. Evidence for a convergent slowdown in primate molecular rates and its implications for the timing of early primate evolution. Proc. Natl Acad. Sci. USA $109,6006-6011$.

Underwood, L., 1894. Notes on our Hepaticae. II. The genus Riccia. Bot. Gaz. 19, 273-278.

Villarreal, J., Crandall-Stotler, B., Hart, M., Long, D., Forrest, L., 2016. Divergence times and the evolution of morphological complexity in an early land plant lineage (Marchantiopsida) with a slow molecular rate. New Phytol. 209, 1734-1746.

Wahlberg, N., Braby, M.F., Brower, A.V., deJong, R., Lee, M.M., Nylin, S., Pierce, N.E., Sperling, F.A., Vila, R., Warren, A.D., Zakharov, E., 2005. Synergistic effects of combining morphological and molecular data in resolving the phylogeny of butterflies and skippers. Proc. R. Soc. B 272, 1577-1586.

Wheeler, J., 1998. Molecular phylogenetic analyses of Riccia and Marchantiales. Doctoral thesis, Oregon State University, 198 pp.

Wheeler, J., 2000. Molecular phylogenetic reconstructions of the marchantioid liverwort radiation. Bryologist 103, 314-333.

Wittlake, E., 1954. The Hepaticae of Arkansas. I. Bryologist 57, 7-18.

Wu, Z.Y., Milne, R.I., Chen, C.J., Liu, J., Wang, H., Li, D.Z., 2015. Ancestral state reconstruction reveals rampant homoplasy of diagnostic morphological characters in Urticaceae, conflicting with current classification schemes. PLoS ONE 10, e0141821.

Yu, Y., Pócs, T., Schäfer-Verwimp, A., Heinrichs, J., Zhu, R., Schneider, H., 2013. Evidence for rampant homoplasy in the phylogeny of the epiphyllous liverwort genus Cololejeunea (Lejeuneaceae). Syst. Bot. 38, 553-563.

\section{Supporting Information}

Additional Supporting Information may be found in the online version of this article:

Fig. S1. Additional support measures.

Fig. S2. Proportion of synapomorphies derived from the sporophyte at each diagnosed branch.

Fig. S3. Strict consensus of trees derived from the ten continuous characters under K3.

Table S1. Primers for sequenced genes.

Table S2. Synapomorphies for each node of the combined tree under block weighting (node numbers in Figure S1).

Table S3. Support values for selected groups. 